\title{
One-loop effective actions and higher spins. Part II
}

\author{
L. Bonora, ${ }^{a}$ M. Cvitan, ${ }^{b}$ P. Dominis Prester, ${ }^{c}$ S.Giaccari ${ }^{b}$ and T. Štemberga ${ }^{b}$ \\ ${ }^{a}$ International School for Advanced Studies (SISSA), and INFN, Sezione di Trieste, \\ Via Bonomea 265, 34136 Trieste, Italy \\ ${ }^{b}$ Department of Physics, Faculty of Science, University of Zagreb, \\ Bijenička cesta 32, 10000 Zagreb, Croatia \\ ${ }^{c}$ Department of Physics, University of Rijeka, \\ Radmile Matejčić 2, 51000 Rijeka, Croatia \\ E-mail: bonora@sissa.it, mcvitan@phy.hr, pprester@phy.uniri.hr, \\ sgiaccari@phy.hr, tstember@phy.hr
}

ABSTRACT: In this paper we continue and improve the analysis of the effective actions obtained by integrating out a scalar and a fermion field coupled to external symmetric sources, started in the previous paper. The first subject we study is the geometrization of the results obtained there, that is we express them in terms of covariant Jacobi tensors. The second subject concerns the treatment of tadpoles and seagull terms in order to implement off-shell covariance in the initial model. The last and by far largest part of the paper is a repository of results concerning all two point correlators (including mixed ones) of symmetric currents of any spin up to 5 and in any dimensions between 3 and 6 . In the massless case we also provide formulas for any spin in any dimension.

Keywords: Higher Spin Gravity, Higher Spin Symmetry, Renormalization Group

ArXiv EPrint: 1709.01738 


\section{Contents}

1 Introduction 1

2 Geometry in effective actions $\quad 3$

2.1 Geometrization in terms of Jacobi tensors 5

3 Tadpoles, seagulls and conservation $\quad 8$

$\begin{array}{llr}3.1 & \text { Fermions - spin } 1 & 10\end{array}$

$\begin{array}{lll}3.1 .1 & \text { Odd parity part } & 12\end{array}$

$\begin{array}{lll}3.2 & \text { Scalars - spin } 1 & 12\end{array}$

$\begin{array}{lll}3.3 & \text { Fermions - spin } 2 & 13\end{array}$

$\begin{array}{lll}3.3 .1 & \text { Even parity part } & 14\end{array}$

$\begin{array}{ll}3.3 .2 \text { Odd parity part } & 16\end{array}$

$\begin{array}{lll}3.4 & \text { Scalars - spin } 2 & 17\end{array}$

4 Correlators $\quad 19$

4.1 Fermion amplitudes for spins $3 \times 3$ and $3 \times 5 \quad 24$

4.2 Expansions in UV and IR for fermions for spins $3 \times 3$ and $3 \times 5 \quad 26$

4.3 All types of correlators 28

$\begin{array}{llr}5 & \text { Conclusion } & 28\end{array}$

$\begin{array}{ll}\text { A Spin 2 - expansions } & 30\end{array}$

B Higher spin traceless actions $\quad 32$

\section{Introduction}

This paper is a follow-up of [1]. In that paper we analyzed the two-point functions of conserved currents of two models (a free scalar and a free Dirac fermion model coupled to diverse backgrounds) in various dimensions. For a background, represented by a completely symmetric field, the two-point function of the current minimally coupled to it is the basic ingredient of its (quadratic) effective action (EA). We found in [1] that the effective action for any background field obtained in this way is based on the corresponding linearized Fronsdal kinetic operator, [2,3], in the nonlocal form introduced by Francia and Sagnotti, $[4,5]$. In view of constructing a covariant action for a completely symmetric tensor field, this result is promising. It suggests that integrating out scalar or fermion fields (or any other field by which one can form conserved currents) may be a useful way to analyze the dynamics of higher spin fields. But of course what we have done in [1] is only the beginning. The crucial next step is the calculation of the three-point functions 
of conserved currents, the analysis of the (lowest order) interaction terms in the effective actions and their consistency with covariance. Before arriving at the three-point functions, it is however necessary to improve our analysis of the quadratic EA. In fact in the course of our research we realized that it inevitably branches out in different directions. At the same time, in [1], several aspects and questions were left behind. In this and a subsequent paper we would like to cover as thoroughly as possible any aspect of the quadratic EA's.

The first issue is the geometrization (at the linear level) of our results in [1]. They were expressed there mostly in terms of a projection operator, which is very convenient in that context because it automatically ensures conservation. But, in this way, the geometrical content of the resulting equations of motion or the EA remains implicit. Now the formulation of our results in terms of geometrical objects is essential, if our target is to arrive at covariant EA's. One first aim of this paper will be to geometrize the results of [1]. We will do it in terms of Jacobi tensors.

A second related important point is related to local subtractions. In [1] we found several violations of the Ward Identities induced by the conservation of the initial current (which induces a gauge invariance of the relevant minimal coupling). Such violations consist of local terms, so that it is rather elementary to recover conservation by subtracting local counterterms from the EA. There is nothing special in this, it is a very ordinary procedure. The interesting point is that it is in general not necessary to do it, because the perturbative field theory formalism already automatically takes care of covariance, provided one takes into account not only the two-point bubble diagrams but also other diagrams such as tadpole and seagull ones. Now, from a practical point of view it is much easier to subtract easily identifiable local counterterms, than calculating additional diagrams to guarantee conservation. The latter could appear as an academic exercise for spin 1 and 2, where we already know the covariant form of the minimal coupling. But, it is important to show that dimensional regularization, which we use, is giving manifestly covariant expressions (without subtractions by hand) as was e.g. done in [6] for scalar matter coupled to gravity. For spin 3 and higher it may be a very useful and even necessary calculation. The reason is that seagull diagrams are related to terms in the initial action that do not belong to the minimal model we start with (a scalar or fermion field minimally coupled to a background field). Conservation (without subtractions) requires the presence of such additional terms and constraints not only their form but also their coefficients. It is clear, that when we consider higher spin backgrounds, this remark may be used in order to determine additional action terms, as well as conditions for their coefficients. This goes in the direction of constructing an initial off shell covariant model, an important target in itself and a necessary step in the construction of a covariant EA.

The third important issue is represented by mixed two-point correlators. In [1] we have considered only two-point functions of each current with itself. Of course this provides basic information about the relevant EA. However higher spin theories are known to be consistent only if they encompass an infinite number of fields (although in $3 \mathrm{~d}$ consistent theories may exist with a finite number of fields). It is obvious that this requires not only the knowledge of the two-point correlator of each higher spin current with itself, but also of any two currents (mixed correlators) coupled to fields that may enter the action. This part has the 
structure of a repository of results about the two point correlators of symmetric currents of spin up to 5 in dimension $3 \leq d \leq 6$ for both the massive scalar and fermion theory. In $3 \mathrm{~d}$ we also consider the odd parity sector which emerges from the parity-breaking fermion mass term, and we find a nice generalization of Pope and Townsend's Chern-Simons-like action in the case when different higher-spin fields are taken into consideration. In the case of equal spins this is the action considered in $[7,8]$ and recently discussed by several authors, see e.g. [9-16].

In this paper we will deal with these three issues. Other topics, such as the discussion of the ambiguities inherent in the choice of the conserved currents in the initial matter model, will be included in a subsequent article. This is a good point to mention that our research is indebted to several preexisting works, in particular with [17-19] as far as the inspiration is concerned, with [20-29] as far as the methods are concerned and with [4, 5, 30-41] for HS theories. Other papers of ours, related to the present one are, beside [1, 42, 43]. For future developments we think that important references are [44-47].

The paper is organized as follows. In the next section we show how to geometrize the results of [1] and of this paper, that is how to express them in terms of Jacobi tensors. In section 3 we discuss the issue of tadpole and seagull terms and how they guarantee covariance without subtractions in the case of spin 1 and 2. Section 4 forms the bulk of the paper. After an explanatory introduction we list all possible conserved two-point correlators for currents up to spin 5, including the mixed ones. This part of the paper (section 4.3) is intended as a source book. Due to its size it is presented in a supplementary material, although several samples of the results are shown also in the main text in sections 4.1-4.2. The results of this part consists of the complete correlators as well as their UV and IR expansions. Several results were already contained in [1]. We have left them here for completeness. Finally, section 5 is devoted to some conclusions.

\section{Geometry in effective actions}

The construction of interacting quantum field theories with massless higher spin $(s>2)$ fields still poses an interesting theoretical problem. On the one hand, there are different "no-go" theorems putting serious constraints on such theories, especially in flat spacetime (see e.g. [48] and references within). On the other hand, we have significant higher spin results: free fields can be constructed in the same manner as in lower spin cases (see, e.g. [49]); a few cubic interaction terms have been constructed in the literature (see [31-38]); most notably, a full consistent covariant HS theory in AdS background has been constructed by Vasilev and collaborators [50-53]. In our previous paper, [1], we remarked that free lower spin field theories possess conserved higher spin currents which simply "beg" to be coupled to higher spin fields. Therefore such simple models seem to be a useful tool to study higher spin theories. A basic ingredient of the approach in [1] is the connection between the on shell conservation of the initial free field theory current and the gauge invariance of the minimal coupling term with the higher spin field, which induces a gauge invariance of the linearized higher spin EA (or covariance of the corresponding equation of motion). In [1] this invariance was left somewhat implicit. There is, however, a way to make it explicit, 
by expressing the results in terms of covariant 'geometric' tensors constructed out of the symmetric higher spin fields. In this section we would like to make connection with such a geometrization program.

In the sequel we first introduce well-known definitions and properties about higher spin tensors, their linearized eom's and their possible geometrical formulations. Then we show how to use this material to express the results obtained in $[1,42]$ and in this paper in a geometric language.

Differences between lower spin $(s \leq 2)$ and higher spin theories emerge already at the level of classical free field theories. The simplest way to construct a free theory of higher spin field is provided by the Fronsdal equation, [2, 3, 54-56]:

$$
\mathcal{F} \equiv \square \varphi-\partial \partial \cdot \varphi+\partial^{2} \varphi^{\prime}=0
$$

where the spin- $s$ field is described by the completely symmetric rank- $s$ tensor field $\varphi \equiv$ $\varphi_{\mu_{1} \cdots \mu_{s}}$. In this expression standard HS conventions from $[4,5,39,40]$ are assumed. ${ }^{1}$ The Fronsdal equation (2.1) is invariant under local transformations parametrised by traceless completely symmetric rank- $(s-1)$ tensor fields $\Lambda \equiv \Lambda_{\mu_{1} \cdots \mu_{s-1}}$

$$
\delta \varphi=\partial \Lambda
$$

with $\Lambda^{\prime}=0$. While this gauge symmetry guarantees that the field propagates only free spin- $s$ excitations, we see that for $s \geq 3$ the gauge symmetry is constrained to tracefree parameters $\Lambda$. One can rewrite the Fronsdal equation in an unconstrained form by introducing a rank- $(s-3)$ compensator field $\alpha$ transforming on (unconstrained) gauge transformations (2.2) as $\delta \alpha=\Lambda^{\prime}$, in the following way

$$
\mathcal{F}=\partial^{3} \alpha
$$

This equation is invariant under the unconstrained gauge transformations (2.2) because the variation of $\alpha$ exactly cancels the variation of the Fronsdal tensor. Most important, the system $\varphi, \alpha$ can be cast in a (local) Lagrangian form. By the partial gauge fixing condition $\alpha=0$ one obtains the original Fronsdal's equation (2.1).

The generalization $\mathcal{F}^{(n)}$ of the Fronsdal differential operator, which is gauge invariant for $n$ large enough, is given in terms of the recursive equation

$$
\mathcal{F}^{(n+1)}=\mathcal{F}^{(n)}+\frac{1}{(n+1)(2 n+1)} \frac{\partial^{2}}{\square} \mathcal{F}^{(n)^{\prime}}-\frac{1}{n+1} \frac{\partial}{\square} \partial \cdot \mathcal{F}^{(n)}
$$

with $\mathcal{F}^{(0)}=\square \varphi$. So, in particular,

$$
\mathcal{F}^{(1)} \equiv \mathcal{F}=\square \varphi-\partial \partial \cdot \varphi+\partial^{2} \varphi^{\prime}
$$

is the original Fronsdal operator. However, the connection with our results cannot be in terms of the tensor $\mathcal{F}^{(n)}$, because the latter does not satisfy a conservation law, while

\footnotetext{
${ }^{1}$ Conventions assume symmetrization over free indices with minimal number of terms and without any symmetry factors. Also, a prime denotes contraction of a pair of indices, so, e.g., $\varphi^{\prime} \equiv \varphi_{\mu_{1} \cdots \mu_{s-2}}=$ $\eta^{\mu_{s-1} \mu_{s}} \varphi_{\mu_{1} \cdots \mu_{s}}$ is a completely symmetric $\operatorname{rank}-(s-2)$ tensor field.
} 
our results are conserved two-point functions. To make the connection one constructs the Einstein-like tensor

$$
\mathcal{G}^{(n)}=\sum_{p=0}^{n}(-1)^{p} \frac{(n-p) !}{2^{p} n !} \eta^{p} \mathcal{F}^{(n)[p]}
$$

where the superscript in square bracket denotes the number of time $\mathcal{F}^{(n)}$ has been traced, and $\eta$ is the Minkowski metric. The association of $\varphi$ with the spin $s$ is as follows:

$$
\left\{\begin{array}{ccc}
s=2 n & s & \text { even } \\
s=2 n-1 & s & \text { odd }
\end{array}\right.
$$

The $\mathcal{G}^{(n)}$ tensor is divergenceless

$$
\partial \cdot \mathcal{G}^{(n)}=0
$$

The free (unconstrained) linearized equations of motion for $\varphi$ are

$$
\mathcal{G}^{(n)}=0
$$

Once again, it can be shown that such an equation can be cast in local Lagrangian form, provided one introduces auxiliary fields (compensators). $\mathcal{G}^{(n)}$ are the objects that can be directly connected with the l.h.s. of (2.15) below.

\subsection{Geometrization in terms of Jacobi tensors}

In [1] all the two-point correlators and corresponding effective actions are presented in momentum space and expressed in terms of the projector

$$
\pi_{\mu \nu}^{(k)}=\eta_{\mu \nu}-\frac{k_{\mu} k_{\nu}}{k^{2}}
$$

Applied to $k^{\nu}$ gives 0, so any two-point function expressed in terms of it alone is conserved. We showed that any conserved correlator for spin $s$ can be written in terms of the following structures:

$$
\begin{aligned}
\tilde{A}_{0}^{(s)}\left(k, n_{1}, n_{2}\right)= & \left(n_{1} \cdot \pi^{(k)} \cdot n_{2}\right)^{s} \\
\tilde{A}_{1}^{(s)}\left(k, n_{1}, n_{2}\right)= & \left(n_{1} \cdot \pi^{(k)} \cdot n_{2}\right)^{s-2}\left(n_{1} \cdot \pi^{(k)} \cdot n_{1}\right)\left(n_{2} \cdot \pi^{(k)} \cdot n_{2}\right) \\
\ldots & \ldots \ldots \\
\tilde{A}_{l}^{(s)}\left(k, n_{1}, n_{2}\right)= & \left(n_{1} \cdot \pi^{(k)} \cdot n_{2}\right)^{s-2 l}\left(n_{1} \cdot \pi^{(k)} \cdot n_{1}\right)^{l}\left(n_{2} \cdot \pi^{(k)} \cdot n_{2}\right)^{l}
\end{aligned}
$$

where $n_{1}, n_{2}$ are generic polarization vectors, and $n_{1} \cdot \pi^{(k)} \cdot n_{2}=n_{1}^{\mu} \pi_{\mu \nu}^{(k)} n_{2}^{\nu}$. There are $\lfloor s / 2\rfloor$ independent such terms. The generic term in the final formulas are combinations of $\tilde{A}_{l}^{(s)}\left(k, n_{1}, n_{2}\right)$ with numerical coefficients $a_{l}$, say

$$
\tilde{E}^{(s)}\left(k, n_{1}, n_{2}\right)=\sum_{l=0}^{\lfloor s / 2\rfloor} a_{l} \tilde{A}_{l}^{(s)}\left(k, n_{1}, n_{2}\right)
$$


preceded by a function of $f(|k|, m)$ and the mass $m .^{2}$ Eq. (2.13) can be easily translated into a corresponding differential operator by Fourier anti-transforming

$$
E^{(s)}\left(\partial, n_{1}, n_{2}\right)=\sum_{l=0}^{\lfloor s / 2\rfloor} a_{l} A_{l}^{(s)}\left(\partial, n_{1}, n_{2}\right)
$$

These are the types of differential operators that appear in the EA's acting on the spin $s$ field $\varphi_{\mu_{1} \ldots \mu_{s}}$. The corresponding eom will take the following form. Set $\left(m^{s} \cdot \varphi\right)=\frac{1}{s !} m_{\mu_{1} \ldots \mu_{s}} \varphi^{\mu_{1} \ldots \mu_{s}}$ and $n_{1}=n \equiv\left\{n_{\mu}\right\}, n_{2}=\partial_{m} \equiv\left\{\frac{\partial}{\partial m_{\mu}}\right\}$. The eom are

$$
\frac{1}{s !}\left(\partial_{n}\right)^{s} E^{(s)}\left(\partial, n, \partial_{m}\right)\left(m^{s} \cdot \varphi\right)=0
$$

multiplied by a function of $k^{2}$ and $m$.

The purpose of this section is to rewrite the equations such as (2.15) in the geometrical form of $[4,5]$.

To this end let us introduce the symbol of $\mathcal{G}^{(n)}, \tilde{\mathcal{G}}^{(n)}\left(k, n_{1}, n_{2}\right)$, as follows. First we saturate all its $s$ naked indices of $\mathcal{G}^{(n)}$ with $n_{1}$ polarizations, then we Fourier transform it and replace the Fourier transform of $\varphi, \tilde{\varphi}$, with a symmetric tensor made out of the product of $s$ polarizations $n_{2}$. Finally we define

$$
\mathcal{G}^{(n)} \equiv \frac{1}{s !}\left(\partial_{n}\right)^{s} \mathcal{G}^{(n)}\left(\partial, n, \partial_{m}\right)\left(m^{s} \cdot \varphi\right)
$$

Then the connection between (2.8) and (2.15) is given by

$$
\frac{1}{k^{2}} \tilde{\mathcal{G}}^{(n)}\left(k, n_{1}, n_{2}\right)=\sum_{l=0}^{\lfloor s / 2\rfloor}(-1)^{l}\left(\begin{array}{c}
\lfloor s / 2\rfloor \\
l
\end{array}\right) \tilde{A}_{l}^{(s)}\left(k, n_{1}, n_{2}\right),
$$

which corresponds to a particular choice of the coefficients $a_{l}$ in (2.13).

Of course we are interested not only in the relation (2.17), but in expressing all the $\tilde{A}_{l}^{(s)}\left(k, n_{1}, n_{2}\right)$ in terms of the $\tilde{\mathcal{G}}^{(n)}\left(k, n_{1}, n_{2}\right)$. To do so we have to take the successive traces of (2.17). We have, for instance

$$
\tilde{\mathcal{G}}^{(n) \prime}=-2\lfloor s / 2\rfloor(2\lfloor s / 2\rfloor+D-4) \tilde{\mathcal{G}}^{(n-1)}\left(n_{2} \cdot \pi^{(k)} \cdot n_{2}\right)
$$

In general

$$
\tilde{\mathcal{G}}^{(n)[p]}=(-2)^{p} \frac{(2\lfloor s / 2\rfloor+D-4) ! !(\lfloor s / 2\rfloor) !}{(2\lfloor s / 2\rfloor+D-2 p-4) ! !(\lfloor s / 2\rfloor-p) !} \tilde{\mathcal{G}}^{(n-p)}\left(n_{2} \cdot \pi^{(k)} \cdot n_{2}\right)^{p}
$$

and

$$
\tilde{\mathcal{G}}^{(n)[n]}=(-2)^{n} \frac{(2\lfloor s / 2\rfloor+D-4) ! !(\lfloor s / 2\rfloor) !}{(D-4) ! !} \tilde{\mathcal{G}}^{(0)}\left(n_{2} \cdot \pi^{(k)} \cdot n_{2}\right)^{n}
$$

\footnotetext{
${ }^{2}$ This function can be expanded in series of $m /|k|$ or $|k| / m$ near the IR and UV, respectively, which gives the tomographic expansions considered in [1]. The latter clearly show that the structures of the two-point functions (and corresponding linearized EA's) are determined by the unique Fronsdal operator appropriate for the given source, although, generally, the operator appears in a nonlocal form and in different gauges. In this paper we consider only these operators and disregard the function $f$.
} 
for $s$ even, with $\tilde{\mathcal{G}}^{(0)}=k^{2}$, and

$$
\tilde{\mathcal{G}}^{(n)[n-1]}=(-2)^{n-1} \frac{(2\lfloor s / 2\rfloor+D-4) ! !(\lfloor s / 2\rfloor) !}{(D-4) ! !} \tilde{\mathcal{G}}^{(1)}\left(n_{2} \cdot \pi^{(k)} \cdot n_{2}\right)^{n-1}
$$

for $s$ odd, with $\tilde{\mathcal{G}}^{(1)}=k^{2}\left(n_{1} \cdot \pi^{(k)} \cdot n_{2}\right)$.

Now, using (2.17), one can write

$$
\begin{aligned}
\left(n_{1} \cdot \pi^{(k)} \cdot n_{2}\right)^{s} \equiv & \tilde{A}_{0}^{(s)}\left(k, n_{1}, n_{2}\right)=\frac{1}{k^{2}} \tilde{\mathcal{G}}^{(n)}\left(k, n_{1}, n_{2}\right) \\
& +\sum_{l=0}^{\lfloor s / 2\rfloor-1}(-1)^{l}\left(\begin{array}{l}
\lfloor s / 2\rfloor \\
l+1
\end{array}\right)\left(n_{1} \cdot \pi^{(k)} \cdot n_{1}\right)^{l+1}\left(n_{1} \cdot \pi^{(k)} \cdot n_{2}\right)^{s-2 l-2}\left(n_{2} \cdot \pi^{(k)} \cdot n_{2}\right)^{l+1}
\end{aligned}
$$

for even $s$, and a similar expression for odd $s$. Now the strategy consists in repeating the same step for the second line in (2.22), by using (2.18) and successively (2.20). The end result is

$$
k^{2}\left(n_{1} \cdot \pi^{(k)} \cdot n_{2}\right)^{s}=\sum_{p=0}^{\lfloor s / 2\rfloor}\left(-\frac{1}{2}\right)^{p} \frac{(2\lfloor s / 2\rfloor+D-2 p-4) ! !}{p !(2\lfloor s / 2\rfloor+D-4) ! !}\left(n_{1} \cdot \pi^{(k)} \cdot n_{1}\right)^{p} \tilde{\mathcal{G}}^{(n)[p]}\left(k, n_{1}, n_{2}\right)
$$

In a similar way one can obtain

$$
\begin{aligned}
& k^{2}\left(n_{1} \cdot \pi^{(k)} \cdot n_{2}\right)^{s-2 l}\left(n_{1} \cdot \pi^{(k)} \cdot n_{1}\right)^{l}\left(n_{2} \cdot \pi^{(k)} \cdot n_{2}\right)^{l} \\
& =\frac{1}{\left(\begin{array}{c}
\lfloor s / 2\rfloor \\
l
\end{array}\right)} \sum_{p=l}^{\lfloor s / 2\rfloor}\left(-\frac{1}{2}\right)^{p}\left(\begin{array}{c}
p \\
l
\end{array}\right) \frac{(2\lfloor s / 2\rfloor+D-2 p-4) ! !}{p !(2\lfloor s / 2\rfloor+D-4) ! !}\left(n_{1} \cdot \pi^{(k)} \cdot n_{1}\right)^{p} \tilde{\mathcal{G}}^{(n)[p]}\left(k, n_{1}, n_{2}\right)
\end{aligned}
$$

In conclusion any expression of the type (2.13), i.e. any conserved structure, can be expressed in terms of the generalized Einstein symbols $\tilde{\mathcal{G}}^{(n)}\left(k, n_{1}, n_{2}\right)$ and its traces. Thus any EA (or any eom) we obtain from our models, by integrating out matter, can be expressed in terms of the generalized Einstein tensor $\mathcal{G}^{(n)}$ and its traces preceded by a function of $\square$ and the mass $m$ of the model, with suitable multiples of the operator

$$
\eta^{\mu \nu}-\frac{\partial^{\mu} \partial^{\nu}}{\square}
$$

acting on the traces. Using (2.6) one can replace the dependence on $\mathcal{G}^{(n)}$ of such expressions with the dependence on $\mathcal{F}^{(n)}$. The geometrization program can be completed by introducing the Jacobi tensors $\mathcal{R}_{\mu_{1}, \ldots \mu_{s} \nu_{1} \ldots \nu_{s}}$ (one of the possible generalizations of the $4 \mathrm{~d}$ Riemann tensor, $[7,57])$ by means of

$$
\frac{1}{(s !)^{2}}\left(m^{s} \cdot \mathcal{R}^{(s)} \cdot n^{s}\right)=\sum_{l=0}^{s} \frac{(-1)^{l}}{s !(s-l) ! l !}(m \cdot \partial)^{s-l}(n \cdot \partial)^{l}\left(m^{l} \cdot \varphi \cdot n^{s-l}\right)
$$

The tensors $\mathcal{R}^{(s)}$ are connected to the $\mathcal{F}^{(n)}$ as follows:

$$
\mathcal{F}^{(n)}= \begin{cases}\frac{1}{\square^{n-1}} \mathcal{R}^{(s)[n]} & s=2 n \\ \frac{1}{\square^{n-1}} \partial \cdot \mathcal{R}^{(s)[n-1]} & s=2 n-1\end{cases}
$$


where the traces in square brackets refer to the first set of indices. In this way we can express any EA or any eom in terms of $\mathcal{R}^{(s)}$ and traces (in the second set of indices) thereof. Further formulations of eoms that are local and include mixed symmetry cases can be found in $[58,59]$.

Since above we have referred to $[4,5]$, we feel that, to end this section, it is opportune for us to clarify the context in which our results are derived and point out the differences with the spirit of $[4,5,39,40]$. In these papers the initial purpose was to write down a generalization of the Fronsdal equations for higher spin in such a way as to avoid the constraints needed in the original formulation of $[2,3]$. The authors of $[4,5]$ chose to sacrifice locality in favor of an unconstrained gauge symmetry. The typical (linearized) non-local equation of motion one obtains in this way is (2.8). As we have already pointed out, it can be shown that such an equation can be cast in Lagrangian form, provided one introduces auxiliary fields (compensators). Therefore one can say that the nonlocality of (2.8) is a gauge artifact, with no physical implication. However equations of motion invariant under unrestricted gauge symmetry are far from unique. There actually exist several families of them depending on arbitrary parameters (by the way, this is evident by reversing the argument above and starting from the generic operator (2.14), instead of the completely fixed one (2.17)). These are all equally valid as long as the field $\varphi$ is considered in isolation and the linearized eom is the free one, (2.8). However, if the spin $s$ system is minimally coupled to a conserved current the question arises as to whether the propagating degrees of freedom are the truly physical ones, i.e. those corresponding to the appropriate little group representation for massless fields. The authors of $[39,40]$ were able to prove that there exist only one choice for the Einstein-like tensor which is Lagrangian and satisfies such a physicality condition.

Such 'physical' Einstein tensors do not correspond, in general, to the kinetic operators we find in our effective action in section 4 below. This is not surprising, as our main goal is covariance: our purpose is to arrive at a covariant EA with respect to a completely unfolded gauge symmetry. In a logical development the next step will be to introduce auxiliary fields to eliminate nonlocalities. Following this we would need to gauge-fix the action and introduce appropriate ghosts to produce the physical propagators. At that point would the problem handled by [39, 40] come to the surface. However, we would like to recall that our immediate prospect is to construct the linearized covariant EA in preparation for the analysis of the three-point function.

\section{Tadpoles, seagulls and conservation}

In this section we wish to illustrate the role of tadpole and seagull diagrams in implementing conservation in two-point correlators. In [1], in order to evaluate the two point correlators of conserved currents we computed only the bubble diagrams formed by two internal scalar or fermion lines and two vertices. In this way we found several violations of the relevant Ward identities. Such violations consist of local terms, so that it was rather elementary to recover conservation by subtracting local counterterms from the EA. However it is in general not necessary to do this, because the perturbative field theory formalism already 
automatically takes care of it provided one takes into account not only the two-point bubble diagrams but also other diagrams such as tadpole and seagull ones, [60,61]. Although this is a rather well-known fact, we would like to show it in detail here for spin 1 and 2 as a guide for the more challenging higher spin cases. The reason is that seagull diagrams reflect the presence in the initial action of additional terms, additional with respect to the minimal couplings (symbolically $\int j \varphi$ ), which are on-shell covariant, but off-shell noncovariant. One of the crucial steps in our program is clearly implementing off-shell gauge covariance of the initial models, that is adding to the minimal couplings in the relevant actions the terms that render them off-shell covariant, at least to the lowest order in a perturbative approach to the gauge symmetry. We know such additional terms exactly in the case of spin 1 and spin 2 (because we know the full covariant action), but not yet for higher spins. In the latter cases, however, we can implement off-shell current conservation by satisfying the corresponding two-point function Ward identity. In turn this requires considering tadpoles and seagull terms. The latter, in particular, originate from the above additional terms, which in this way may hopefully be identified. ${ }^{3}$

Hereafter in this section we work out the cases of spin 1 and spin 2 in any dimension (in $3 \mathrm{~d}$ for the odd parity part) in detail, showing the role of tadpole and seagull terms in the Ward identities for two-point functions of spin 1 and 2 respectively, and their origin in the various terms of the initial actions. We keep the derivation at a pedagogical level and, for completeness, we analyze the full structure of the relevant two-point functions and, in particular, their IR and UV expansions, as well as their contributions to the EA's.

Starting from the generating function

$$
Z[a]=e^{i W[a]}=\int D \psi D \bar{\psi} e^{i\left(S_{0}+S_{\mathrm{int}}[a]\right)}
$$

where $a$ is the external higher spin field, we will compute the effective action for the external source fields up to the quadratic order:

$$
\begin{aligned}
i W[a]= & i W[0]+\int d^{d} x a_{\mu_{1} \ldots \mu_{s}}(x) \Theta^{\mu_{1} \ldots \mu_{s}}(x) \\
& +\frac{1}{2 !} \int d^{d} x d^{d} y a_{\mu_{1} \ldots \mu_{s}}(x) a_{\nu_{1} \ldots \nu_{s}}(y) T^{\mu_{1} \ldots \mu_{s} \nu_{1} \ldots \nu_{s}}(x, y)+\ldots
\end{aligned}
$$

where

$$
\Theta^{\mu_{1} \ldots \mu_{s}}(x)=\left.\frac{\delta(i W[a])}{\delta a_{\mu_{1} \ldots \mu_{s}}(x)}\right|_{a=0}
$$

is a tadpole (1-point function) and

$$
T^{\mu_{1} \ldots \mu_{s} \nu_{1} \ldots \nu_{s}}(x, y)=\left.\frac{\delta^{2}(i W[a])}{\delta a_{\mu_{1} \ldots \mu_{s}}(x) \delta a_{\nu_{1} \ldots \nu_{s}}(y)}\right|_{a=0}
$$

\footnotetext{
${ }^{3} \mathrm{An}$ approach related to ours is outlined in $[24,25]$. It is based on Weyl quantization. Its main advantage is that it provides a full quantum action and quantum symmetry for the initial scalar model. It will be interesting to compare the two approaches.
} 
is a 2-point function. Using Feynman diagrams we wish to compute the 2-point function including not only the bubble diagram (as in [1]) but also tadpoles and seagulls.

The one-loop 1-pt correlator for the external field is (up to the linear order):

$$
\begin{aligned}
\left\langle\left\langle J^{\mu_{1} \ldots \mu_{s}}(x)\right\rangle\right\rangle & =\frac{\delta W[a]}{\delta a_{\mu_{1} \ldots \mu_{s}}(x)} \\
& =-i\left(\Theta^{\mu_{1} \ldots \mu_{s}}(x)+\int d^{d} y a_{\nu_{1} \ldots \nu_{s}}(y) T^{\mu_{1} \ldots \mu_{s} \nu_{1} \ldots \nu_{s}}(x, y)+\ldots\right)
\end{aligned}
$$

External spin $s$ fields $a_{\mu_{1} \ldots \mu_{s}}$ are in particular

$$
\begin{array}{lrlrl}
\text { Spin 1 } & a_{\mu}=A_{\mu} & & \text { gauge field } \\
\text { Spin 2 } & a_{\mu \nu}=h_{\mu \nu} & & \text { graviton field }
\end{array}
$$

We will need one-loop conservation which for spin 1 reads

$$
\partial_{\mu}\left\langle\left\langle J^{\mu}(x)\right\rangle\right\rangle=0
$$

The Ward identity for the two-point function in momentum space can be written as

$$
k_{\mu} \tilde{T}^{\mu \nu}(k)=0
$$

Furthermore, for spin 2, the energy-momentum tensor is defined with $\left\langle\left\langle T^{\mu \nu}(x)\right\rangle\right\rangle=$ $\frac{2}{\sqrt{g}} \frac{\delta W}{\delta h_{\mu \nu}(x)}$. The full conservation law of the energy-momentum tensor is

$$
\nabla_{\mu}\left\langle\left\langle T^{\mu \mu}(x)\right\rangle\right\rangle=0
$$

Hence, the Ward identity for one-point function is

$$
\partial_{\mu} \Theta^{\mu \mu}(x)=0
$$

while for two-point correlator we have

$$
\begin{aligned}
\partial_{\mu} T^{\mu \mu \nu \nu}(x, y)= & \frac{1}{2} \eta^{\nu \nu} \delta(x-y) \partial_{\mu} \Theta^{\mu \mu}(x)+\frac{1}{2} \Theta^{\nu \nu}(x) \partial^{\mu} \delta(x-y) \\
& -\partial_{\mu}\left(\delta(x-y) \Theta^{\mu \nu}(x)\right) \eta^{\mu \nu}
\end{aligned}
$$

As we will see, the tadpole contribution is $\tilde{\Theta}^{\mu \mu}(k)=\tilde{\Theta} \eta^{\mu \mu}$ where $\tilde{\Theta}$ is a constant. The Ward identity in momentum space is now

$$
k_{\mu} \tilde{T}^{\mu \mu \nu \nu}(k)=\left[-k^{\nu} \eta^{\mu \nu}+\frac{1}{2} k^{\mu} \eta^{\nu \nu}\right] \tilde{\Theta}
$$

\subsection{Fermions - spin 1}

The action for the theory of fermions interacting with gauge field can be written as

$$
S=\int \mathrm{d} x\left[\bar{\psi}\left(i \gamma^{\mu} D_{\mu}-m\right) \psi\right]
$$


where $D_{\mu}=\partial_{\mu}-i A_{\mu}$. There is one fermion-fermion-photon vertex

$$
V_{f f p}^{\mu}: i \gamma^{\mu}
$$

In the case of fermions coupled to gauge field the tadpole diagram vanishes, while the seagull is zero because the theory is linear in the gauge field. The only contribution we get from the 2-pt correlator ((11.7) from [1]) which in the momentum space reads

$$
\begin{aligned}
\tilde{T}^{\mu \nu}(k)= & \frac{2^{-d+\left\lfloor\frac{d}{2}\right\rfloor} i \pi^{-\frac{d}{2}} m^{d-2}}{4 m^{2}-k^{2}} \Gamma\left(1-\frac{d}{2}\right) \\
& \times\left(-4 m^{2}+{ }_{2} F_{1}\left[1,1-\frac{d}{2} ; \frac{3}{2} ; \frac{k^{2}}{4 m^{2}}\right]\left(4 m^{2}+(d-2) k^{2}\right)\right) \pi^{\mu \nu}
\end{aligned}
$$

where $\pi^{\mu \nu}=\eta^{\mu \nu}-\frac{k^{\mu} k^{\nu}}{k^{2}}$ is the projector. Since the 2-point correlator can be expressed in terms of the projector, it satisfies Ward identity (3.7) We can expand the two-point correlator in the IR region

$$
\tilde{T}^{\mu \nu}(k)=-2^{1-d+\left\lfloor\frac{d}{2}\right\rfloor} i m^{d-2} \pi^{-\frac{d}{2}} \sum_{n=1}^{\infty} \frac{n m^{-2 n} \Gamma\left(2 n-\frac{d}{2}\right)}{2^{n}(2 n+1) ! !} k^{2 n} \pi^{\mu \nu}
$$

Using the Fourier transform of (3.16) in the one-loop 1-point function (3.5) we get

$$
\left\langle\left\langle J^{\mu}(x)\right\rangle\right\rangle=2^{1-d+\left\lfloor\frac{d}{2}\right\rfloor} m^{d-2} \pi^{-\frac{d}{2}} \sum_{n=1}^{\infty} \frac{(-1)^{n} n m^{-2 n} \Gamma\left(2 n-\frac{d}{2}\right)}{2^{n}(2 n+1) ! !} \square^{n-1} \partial_{\nu} F^{\mu \nu}
$$

The one-loop 1-point correlator satisfies (3.7) Using the same expansion in the IR (3.16) for the effective action (3.2) we obtain

$$
\begin{gathered}
W=2^{-1-d+\left\lfloor\frac{d}{2}\right\rfloor} m^{d-2} \pi^{-\frac{d}{2}} \sum_{n=1}^{\infty} \frac{(-1)^{n} n m^{-2 n} \Gamma\left(2 n-\frac{d}{2}\right)}{2^{n}(2 n+1) ! !} \int \mathrm{d}^{d} x F_{\mu \nu} \square^{n-1} F^{\mu \nu} \\
\stackrel{\mathrm{IR}}{=}-\frac{2^{-2-d+\left\lfloor\frac{d}{2}\right\rfloor}}{3} m^{d-4} \pi^{-\frac{d}{2}} \Gamma\left(2-\frac{d}{2}\right) \int \mathrm{d}^{d} x F_{\mu \nu} F^{\mu \nu}
\end{gathered}
$$

So, in the IR region (large $\mathrm{m}$ ) we get the Maxwell action.

Furthermore, the dominating term in the UV $\left(O\left(m^{0}\right)\right)$ of $(3.15)$ corresponds to the massless case (B.2) from [1]

$$
\tilde{T}^{\mu \nu}(k) \stackrel{\mathrm{UV}}{=}-\frac{2^{2-2 d+\left\lfloor\frac{d}{2}\right\rfloor} \pi^{\frac{3}{2}-\frac{d}{2}}(d-2)}{\left(-1+e^{i \pi d}\right) \Gamma\left(\frac{d+1}{2}\right)}\left(k^{2}\right)^{\frac{d}{2}-1} \pi^{\mu \nu}
$$

The effective action in the UV is then

$$
W \stackrel{\mathrm{UV}}{=} \frac{(-1)^{\frac{d}{2}} 2^{1-2 d+\left\lfloor\frac{d}{2}\right\rfloor} \pi^{\frac{3}{2}-\frac{d}{2}}(d-2)}{\left(-1+e^{i \pi d}\right) \Gamma\left(\frac{d+1}{2}\right)} F^{\mu \nu} \square^{\frac{d}{2}-2} F_{\mu \nu}
$$




\subsubsection{Odd parity part}

For the analysis of the odd parity correlators we will restrict ourselves to $d=3$. The odd part of the two-point correlator is non-vanishing only in $3 d$ and it is given by

$$
\tilde{T}_{o}^{\mu \nu}(k)=\frac{m}{2 \pi k} \operatorname{Arc} \operatorname{Coth}\left(\frac{2 m}{k}\right) \epsilon^{\mu \nu \lambda} k_{\lambda}
$$

The expansion of (3.21) in the IR reads

$$
\tilde{T}_{o}^{\mu \nu}(k)=\frac{1}{\pi} \sum_{n=0}^{\infty} \frac{k^{2 n} m^{-2 n}}{2^{2(n+1)}(2 n+1)} \epsilon^{\mu \nu \lambda} k_{\lambda}
$$

Using the IR expansion in (3.5), the odd part of the one-loop 1-point correlator is now

$$
\left\langle\left\langle J^{\mu}(x)\right\rangle\right\rangle=\frac{1}{\pi} \sum_{n=0}^{\infty} \frac{(-1)^{n} m^{-2 n}}{2^{2 n+3}(2 n+1)} \epsilon^{\mu \nu \lambda} \square^{n} F_{\lambda \nu}
$$

and just like the even parity part satisfies (3.7). The effective action in the IR (the dominating term)

$$
W \stackrel{\text { IR }}{=} \frac{1}{8 \pi} \epsilon^{\mu \nu \lambda} \int d^{3} x A_{\mu} \partial_{\nu} A_{\lambda}+\ldots
$$

corresponds to Chern-Simons term in 3d

$$
S_{\mathrm{CS}}=\frac{1}{8 \pi} \int d^{3} x \operatorname{Tr}\left(A \wedge d A+\frac{2}{3} A \wedge A \wedge A\right)
$$

\subsection{Scalars - spin 1}

The action in the scalar QED model is

$$
S=\int \mathrm{d}^{d} x\left[D_{\mu} \varphi^{\dagger} D^{\mu} \varphi-m^{2} \varphi^{\dagger} \varphi\right]
$$

where $D_{\mu}=\partial_{\mu}-i A_{\mu}$. The full covariant action is

$$
S=\int \mathrm{d} x\left[\partial_{\mu} \varphi^{\dagger} \partial^{\mu} \varphi+i A_{\mu}\left(\varphi^{\dagger} \partial^{\mu} \varphi-\partial^{\mu} \varphi^{\dagger} \varphi\right)+A_{\mu} A^{\mu} \varphi^{\dagger} \varphi-m^{2} \varphi^{\dagger} \varphi\right]
$$

In the scalar model the scalar-scalar-photon vertex is

$$
V_{s s p}^{\mu}\left(p, p^{\prime}\right):-i\left(p+p^{\prime}\right)^{\mu}
$$

and we also have scalar-scalar-photon-photon vertex (coming from $\int d^{d} x A^{\mu} A_{\mu} \varphi^{\dagger} \varphi$ term in Lagrangian)

$$
V_{s s p p}^{\mu \nu}\left(p, p^{\prime}\right): 2 i \eta^{\mu \nu}
$$

The two-point function for the massive scalar in any dimension $d$ for spin $s=1$ is

$$
\tilde{T}^{\mu \nu}(k)=-2^{1-d} i \pi^{-d / 2} m^{d-2} \Gamma\left(1-\frac{d}{2}\right)\left({ }_{2} F_{1}\left[1,1-\frac{d}{2} ; \frac{3}{2} ; \frac{k^{2}}{4 m^{2}}\right] \pi^{\mu \nu}+\frac{k^{\mu} k^{\nu}}{k^{2}}\right)
$$


which has a non-conserved part. However, since the theory is quadratic in the external photon field $A$ we also have a seagull diagram (which is obtained by joining with a unique a fermion line the two fermion legs of the vertex (3.29)) for which we obtain

$$
\tilde{T}_{(s)}^{\mu \nu}(k)=2^{1-d} i \pi^{-\frac{d}{2}} m^{d-2} \Gamma\left(1-\frac{d}{2}\right) \eta^{\mu \nu}
$$

After combining (3.30) and (3.31) we can write down the full 2-point function

$$
\tilde{T}^{\mu \nu}(k)=2^{1-d} i \pi^{-\frac{d}{2}} m^{d-2} \Gamma\left(1-\frac{d}{2}\right)\left(1-{ }_{2} F_{1}\left[1,1-\frac{d}{2} ; \frac{3}{2} ; \frac{k^{2}}{4 m^{2}}\right]\right) \pi^{\mu \nu},
$$

which is conserved.

Expanding the two-point function (3.32) in the IR gives

$$
\tilde{T}^{\mu \nu}(k)=-2^{-d} i m^{d-4} \pi^{-\frac{d}{2}} \sum_{n=0}^{\infty} \frac{m^{-2 n} \Gamma\left(2+n-\frac{d}{2}\right)}{2^{n}(2 n+3) ! !} k^{2 n+2} \pi^{\mu \nu}
$$

Using the IR expansion together with (3.5), the one-loop 1-point function (3.5) now reads

$$
\left\langle\left\langle J^{\mu}\right\rangle\right\rangle=-2^{-d} m^{d-4} \pi^{-\frac{d}{2}} \sum_{n=0}^{\infty} \frac{(-1)^{n} m^{-2 n} \Gamma\left(2+n-\frac{d}{2}\right)}{2^{n}(2 n+3) ! !} \square^{n} \partial_{\nu} F^{\mu \nu}
$$

On the other hand, the dominating term of the effective action in the IR region is

$$
W \stackrel{\mathrm{IR}}{=}-\frac{2^{-d}}{3} m^{d-4} \pi^{-\frac{d}{2}} \Gamma\left(2-\frac{d}{2}\right) \int \mathrm{d}^{d} x F_{\mu \nu} F^{\mu \nu}
$$

In the IR (for large mass $\mathrm{m}$ ) we get the Maxwell action.

The leading order term of the expansion in the UV (term $m^{0}$ corresponds to (B.13) from [1])

$$
\tilde{T}^{\mu \nu}(k) \stackrel{\mathrm{UV}}{=}-\frac{2^{3-2 d} \pi^{\frac{3}{2}-\frac{d}{2}}\left(k^{2}\right)^{\frac{d}{2}-1}}{\left(-1+e^{i \pi d}\right) \Gamma\left(\frac{d+1}{2}\right)} \pi^{\mu \nu}
$$

Hence, the effective action in the UV is

$$
W \stackrel{\mathrm{UV}}{=}-i \frac{(-1)^{\frac{d}{2}} 2^{3-2 d} \pi^{\frac{3}{2}-\frac{d}{2}}}{\left(-1+e^{i \pi d}\right) \Gamma\left(\frac{d+1}{2}\right)} \int d^{d} x F^{\mu \nu} \square^{\frac{d}{2}-2} F_{\mu \nu}
$$

\subsection{Fermions - spin 2}

Let us consider the free fermion theory in a generic dimension $d$

$$
S=\int d^{d} x \sqrt{|g|}\left[i \bar{\psi} E_{a}^{m} \gamma^{a}\left(\partial_{m}+\frac{1}{2} \Omega_{m}\right) \psi-m \bar{\psi} \psi\right]
$$

where $E_{a}^{m}$ is the inverse vierbein. From now on we will set $g_{\mu \nu}=\eta_{\mu \nu}+h_{\mu \nu}$. Using the following expansions

$$
\begin{aligned}
g^{\mu \nu} & =\eta^{\mu \nu}-h^{\mu \nu}+\left(h^{2}\right)^{\mu \nu}+\ldots, & \sqrt{|g|} & =1+\frac{1}{2} h+\frac{1}{8} h^{2}-\frac{1}{4} h^{\mu \nu} h_{\mu \nu}+\ldots, \\
e_{a}^{\mu} & =\delta_{a}^{\mu}-\frac{1}{2} h_{a}^{\mu}+\frac{3}{8}\left(h^{2}\right)_{a}^{\mu}+\ldots, & e_{\mu}^{a} & =\delta_{\mu}^{a}+\frac{1}{2} h_{\mu}^{a}-\frac{1}{8}\left(h^{2}\right)_{\mu}^{a}+\ldots
\end{aligned}
$$


we can expand the parity even part of the action (3.38) in powers of $h$ :

$$
\begin{aligned}
S_{e}= & \int d^{d} x\left[\frac{i}{2} \bar{\psi} \gamma^{m} \stackrel{\leftrightarrow}{\partial}_{m} \psi-m \bar{\psi} \psi+\frac{1}{2} h_{\mu}^{\mu}\left(\frac{i}{2} \bar{\psi} \gamma^{m} \stackrel{\leftrightarrow}{\partial}_{m} \psi-m \bar{\psi} \psi\right)-\frac{i}{4} \bar{\psi} h_{a}^{m} \gamma^{a} \stackrel{\leftrightarrow}{\partial}_{m} \psi\right. \\
& +\frac{1}{8}\left(\left(h_{\mu}^{\mu}\right)^{2}-2 h_{\mu}^{\nu} h_{\nu}^{\mu}\right)\left(\frac{i}{2} \bar{\psi} \gamma^{m} \stackrel{\leftrightarrow}{\partial}_{m} \psi-m \bar{\psi} \psi\right) \\
& \left.-\frac{i}{8} h_{\mu}^{\mu} \bar{\psi} h_{a}^{m} \gamma^{a} \stackrel{\leftrightarrow}{\partial}_{m} \psi+\frac{3 i}{16} \bar{\psi}\left(h^{2}\right)_{a}^{m} \gamma^{a} \stackrel{\leftrightarrow}{\partial}_{m} \psi+\ldots\right]
\end{aligned}
$$

There is one fermion-fermion-graviton vertex: ${ }^{4}$

$$
V_{f f h}^{\mu \mu}\left(p, p^{\prime}\right):-\frac{i}{4}\left(p+p^{\prime}\right)^{\mu} \gamma^{\mu}+\frac{i}{4} \eta^{\mu \mu}\left(\not p+\not p^{\prime \prime}-2 m\right)
$$

and one vertex with two fermions and two gravitons:

$$
\begin{aligned}
V_{f f h h}^{\mu \mu \nu \nu}\left(p, p^{\prime}\right): & \frac{3 i}{16}\left(\left(p+p^{\prime}\right)^{\mu} \gamma^{\nu} \eta^{\mu \nu}+\left(p+p^{\prime}\right)^{\nu} \gamma^{\mu} \eta^{\mu \nu}\right) \\
& +\frac{i}{8}\left(\not p+\not p p^{\prime \prime}-2 m\right)\left(\eta^{\mu \mu} \eta^{\nu \nu}-2 \eta^{\mu \nu} \eta^{\mu \nu}\right) \\
& -\frac{i}{8}\left(\left(p+p^{\prime}\right)^{\mu} \gamma^{\mu} \eta^{\nu \nu}+\left(p+p^{\prime}\right)^{\nu} \gamma^{\nu} \eta^{\mu \mu}\right)
\end{aligned}
$$

We can also expand the odd parity part of the action (the latter contains a part proportional to the completely antisymmetric symbol). We will restrict ourselves to $3 \mathrm{~d}$ because only in this case can we get a non-vanishing contribution to the effective action and 1-point correlator.

$$
S_{o}=\frac{1}{16} \int d^{3} x \epsilon^{a b c} \partial_{a} h_{b \sigma} h_{c}^{\sigma} \bar{\psi} \psi
$$

The relevant vertex with two fermions and two gravitons is

$$
V_{\epsilon, f f h h}^{\mu \mu \nu \nu}: \frac{1}{16} \eta^{\mu \nu} \epsilon^{\mu \nu \lambda}\left(k-k^{\prime}\right)_{\lambda}
$$

\subsubsection{Even parity part}

The tadpole contribution is now

$$
\tilde{\Theta}^{\mu \mu}(k)=-2^{-2-d+\left\lfloor\frac{d}{2}\right\rfloor} i m^{d} \pi^{\frac{d}{2}} \Gamma\left(-\frac{d}{2}\right) \eta^{\mu \mu}=\tilde{\Theta} \eta^{\mu \mu}
$$

where $\tilde{\Theta}$ is a constant. Since the theory of gravity is non-linear we have a contribution from the seagull term, which can be written as

$$
\tilde{T}_{(s)}^{\mu \mu \nu \nu}(k)=2^{-3-d+\left\lfloor\frac{d}{2}\right\rfloor} i m^{d} \pi^{\frac{d}{2}} \Gamma\left(-\frac{d}{2}\right)\left(3 \eta^{\mu \nu} \eta^{\mu \nu}-2 \eta^{\mu \mu} \eta^{\nu \nu}\right)
$$

\footnotetext{
${ }^{4}$ We use the convention according to which two repeated identical indices represent a symmetrized couple of indices, and so on.
} 
The bubble diagram contributes two parts, the transverse (conserved) part,

$$
\begin{aligned}
\tilde{T}_{t}^{\mu \mu \nu \nu}(k)= & -\frac{1}{d(d+1) k^{2}} 2^{-2-d+\left\lfloor\frac{d}{2}\right\rfloor} i m^{d} \pi^{\frac{d}{2}} \Gamma\left(1-\frac{d}{2}\right) \\
& {\left[\left(-8 m^{2}+(d+1) k^{2}+{ }_{2} F_{1}\left[1,-\frac{d}{2}, \frac{1}{2}, \frac{k^{2}}{4 m^{2}}\right]\left(8 m^{2}+(d-1) k^{2}\right)\right) \pi^{\mu \nu} \pi^{\mu \nu}\right.} \\
& \left.+\left(-4 m^{2}+(d+1) k^{2}+{ }_{2} F_{1}\left[1,-\frac{d}{2}, \frac{1}{2}, \frac{k^{2}}{4 m^{2}}\right]\left(4 m^{2}-k^{2}\right)\right) \pi^{\mu \mu} \pi^{\nu \nu}\right]
\end{aligned}
$$

whose expansion in the IR is

$$
\tilde{T}_{t}^{\mu \mu \nu \nu}(k)=-2^{-3-d+\left\lfloor\frac{d}{2}\right\rfloor} i m^{d} \pi^{-\frac{d}{2}} \sum_{n=1}^{\infty} \frac{m^{-2 n} \Gamma\left(n-\frac{d}{2}\right)}{2^{n}(2 n+1) ! !} k^{2 n}\left((2 n-1) \pi^{\mu \nu} \pi^{\mu \nu}-\pi^{\mu \mu} \pi^{\nu \nu}\right),
$$

and the non-transverse (non-conserved) part

$$
\tilde{T}_{n t}^{\mu \mu \nu \nu}(k)=-2^{-3-d+\left\lfloor\frac{d}{2}\right\rfloor} i m^{d} \pi^{\frac{d}{2}} \Gamma\left(-\frac{d}{2}\right)\left(\eta^{\mu \nu} \eta^{\mu \nu}-\eta^{\mu \mu} \eta^{\nu \nu}\right) .
$$

Taking formulas (3.45), (3.46), (3.47) and (3.49) and substituting them in (3.12) we can see that the Ward identity is satisfied for any dimension $d$.

The one-loop 1-point function (energy-momentum tensor) defined as $\left\langle\left\langle T^{\mu \nu}(x)\right\rangle\right\rangle=$ $\frac{2}{\sqrt{g}} \frac{\delta W}{\delta h_{\mu \nu}(x)}$ now becomes

$$
\begin{aligned}
\left\langle\left\langle T^{\mu \mu}(x)\right\rangle\right\rangle= & -2^{-1-d+\left\lfloor\frac{d}{2}\right\rfloor} m^{d} \pi^{-\frac{d}{2}}\left[\Gamma\left(-\frac{d}{2}\right) g^{\mu \mu}+\sum_{n=1}^{\infty} \frac{(-1)^{n} m^{-2 n} \Gamma\left(n-\frac{d}{2}\right)}{2^{n+1}(2 n+1) ! !}\right. \\
& \left.\times\left((2 n-1) \square^{n-1} G^{\mu \mu}+(n-1) \square^{n-2}\left(\eta^{\mu \mu} \square-\partial^{\mu} \partial^{\mu}\right) R\right)\right]+O\left(h^{2}\right)
\end{aligned}
$$

where $G_{\mu \mu}=R_{\mu \mu}-\frac{1}{2} \eta_{\mu \mu} R$ is the Einstein tensor. The energy-momentum tensor is clearly divergence free (3.9). For the effective action in the IR we obtain (in the even parity sector)

$$
\begin{aligned}
W \stackrel{\mathrm{IR}}{=} & -2^{-1-d+\left\lfloor\frac{d}{2}\right\rfloor} m^{d} \pi^{-\frac{d}{2}} \int \mathrm{d}^{d} x \sqrt{g} \times\left[\Gamma\left(-\frac{d}{2}\right)-\frac{\Gamma\left(1-\frac{d}{2}\right)}{24 m^{2}} R\right. \\
& \left.-\frac{\Gamma\left(2-\frac{d}{2}\right)}{80 m^{4}}\left(R_{\mu \nu \lambda \rho} R^{\mu \nu \lambda \rho}-2 R_{\mu \nu} R^{\mu \nu}+\frac{1}{3} R^{2}\right)+\ldots\right]+O\left(h^{3}\right)
\end{aligned}
$$

The divergent part of the effective action for $d=4$ (i.e. $d=4+\varepsilon$ ) is

$$
W \stackrel{\mathrm{IR}}{=} \frac{1}{8 \pi^{2} \varepsilon} \int \mathrm{d}^{4} x \sqrt{g}\left(m^{4}+\frac{1}{12} m^{2} R-\frac{1}{40} \mathcal{W}^{2}+\ldots\right)+O\left(h^{3}\right)
$$

The first term is a cosmological constant term and the second is the linearized EinsteinHilbert action. The third term $\left(m^{0}\right.$ term $)$ is the Weyl density $\mathcal{W}^{2}=R_{\mu \nu \lambda \rho} R^{\mu \nu \lambda \rho}-$ $2 R_{\mu \nu} R^{\mu \nu}+\frac{1}{3} R^{2}$ (conformal invariant in $4 \mathrm{~d}$ ). 
The dominating term in the UV $\left(O\left(m^{0}\right)\right.$ term corresponds to (B.3) from [1]) of the transverse part $\tilde{T}_{t}^{\mu \mu \nu \nu}(k)$ is

$$
\tilde{T}_{t}^{\mu \mu \nu \nu}(k) \stackrel{\mathrm{UV}}{=} \frac{2^{-3-2 d+\left\lfloor\frac{d}{2}\right\rfloor} \pi^{\frac{3}{2}-\frac{d}{2}}\left(k^{2}\right)^{\frac{d}{2}}}{\left(-1+e^{i \pi d}\right) \Gamma\left(\frac{d+3}{2}\right)}\left((d-1) \pi^{\mu \nu} \pi^{\mu \nu}-\pi^{\mu \mu} \pi^{\nu \nu}\right)
$$

The effective action in the UV is then

$$
\begin{aligned}
W \stackrel{\mathrm{UV}}{=} & (-1)^{\frac{d}{2}} \frac{2^{-4-2 d+\left\lfloor\frac{d}{2}\right\rfloor} \pi^{\frac{3}{2}-\frac{d}{2}}}{\left(-1+e^{i \pi d}\right) \Gamma\left(\frac{d+3}{2}\right)} \int \mathrm{d}^{d} x \sqrt{g}\left[(d-4) R_{\mu \nu \lambda \rho} \square^{\frac{d}{2}-2} R^{\mu \nu \lambda \rho}\right. \\
& \left.+6\left(R_{\mu \nu \lambda \rho} \square^{\frac{d}{2}-2} R^{\mu \nu \lambda \rho}-2 R_{\mu \nu} \square^{\frac{d}{2}-2} R^{\mu \nu}+\frac{1}{3} R \square^{\frac{d}{2}-2} R\right)+\ldots\right]+O\left(h^{3}\right)
\end{aligned}
$$

\subsubsection{Odd parity part}

In $3 \mathrm{~d}$ the contribution from the seagull diagram with vertex (3.44) becomes

$$
\tilde{T}_{(s, o)}^{\mu \mu \nu \nu}(k)=-\frac{m^{2}}{16 \pi} \eta^{\mu \nu} \epsilon^{\mu \nu \lambda} k_{\lambda}
$$

The odd part of the two-point correlator is non-vanishing only in $3 d$ (the vertex is (3.41)). The transverse part can be written as

$$
\tilde{T}_{t, o}^{\mu \mu \nu \nu}(k)=-\frac{m}{64 \pi k}\left(\left(k^{2}-4 m^{2}\right) \operatorname{ArcCoth}\left(\frac{2 m}{k}\right)+2 m k\right) \pi^{\mu \nu} \epsilon^{\mu \nu \lambda} k_{\lambda}
$$

and the expansion of $\tilde{T}_{t, o}^{\mu \mu \nu \nu}(k)$ in the IR is

$$
\tilde{T}_{t, o}^{\mu \mu \nu \nu}(k)=-\frac{1}{64 \pi} \sum_{n=0}^{\infty} \frac{k^{2(n+1)} m^{-2 n}}{4^{2 n}\left(4(n+1)^{2}-1\right)} \pi^{\mu \nu} \epsilon^{\mu \nu \lambda} k_{\lambda}
$$

The odd non-transverse part reads ${ }^{5}$

$$
\tilde{T}_{n t, o}^{\mu \mu \nu \nu}(k)=\frac{m^{2}}{16 \pi} \eta^{\mu \nu} \epsilon^{\mu \nu \lambda} k_{\lambda}
$$

and can be canceled by the seagull contribution (3.55). So, only the transverse odd part remains. The odd part of the one-loop 1-pt function (energy-momentum tensor)

$$
\left\langle\left\langle T^{\mu \mu}(x)\right\rangle\right\rangle=\frac{1}{32 \pi} \sum_{n=0}^{\infty} \frac{(-1)^{n} m^{-2 n}}{4^{2 n}\left(4(n+1)^{2}-1\right)} \square^{n} C^{\mu \mu}
$$

where $C^{\mu \mu}$ is linearized the Cotton tensor (A.16). The effective action in the IR (the dominating term)

$$
W \stackrel{\mathrm{IR}}{=}-\frac{1}{384 \pi} \epsilon^{\mu \nu \lambda} \int d^{3} x h_{\nu \nu}\left(\partial_{\lambda} \partial^{\mu} \partial^{\nu} h_{\mu \mu}-\partial_{\lambda} \square h_{\mu}^{\nu}\right)+O\left(h^{3}\right)
$$

corresponds to gravitational Chern-Simons term in 3d

$$
S_{\mathrm{gCS}}=\frac{1}{192 \pi} \epsilon^{\mu \nu \lambda} \int d^{3} x\left(\partial_{\mu} \omega_{\nu}^{a b} \omega_{\lambda}^{b a}+\frac{2}{3} \omega_{\mu a}{ }^{b} \omega_{\nu b}{ }^{c} \omega_{\lambda c}{ }^{a}\right)
$$

\footnotetext{
${ }^{5}$ In the notation from the previous paper $\pi^{\mu \nu} \epsilon^{\mu \nu \lambda} k_{\lambda}$ corresponds to $\left(n_{1} \cdot \pi \cdot n_{2}\right) \epsilon\left(k \cdot n_{1} \cdot n_{2}\right)$.
} 


\subsection{Scalars - spin 2}

Let us consider the action of a scalar field $\varphi$ in a curved space $\left(g_{\mu \nu}=\eta_{\mu \nu}+h_{\mu \nu}\right)$ with a scalar curvature coupling

$$
S=\int d^{d} x \sqrt{g}\left(g^{\mu \nu} \partial_{\mu} \varphi^{\dagger} \partial_{\nu} \varphi-m^{2} \varphi^{\dagger} \varphi+\xi R \varphi^{\dagger} \varphi\right)
$$

Let us redefine $\phi=g^{\frac{1}{4}} \varphi$. The expansion of the action in the external field $\mathrm{h}$ is

$$
\begin{aligned}
S= & \int d^{d} x\left[\eta^{\mu \nu} \partial_{\mu} \phi^{\dagger} \partial_{\nu} \phi-m^{2} \phi^{\dagger} \phi+h^{\mu \nu}\left(\frac{1}{4} \phi^{\dagger} \stackrel{\leftrightarrow}{\partial}_{\mu} \stackrel{\leftrightarrow}{\partial}_{\nu} \phi+\left(\xi-\frac{1}{4}\right)\left(\partial_{\mu} \partial_{\nu}-\square \eta_{\mu \nu}\right) \phi^{\dagger} \phi\right)\right. \\
& +h^{\mu \sigma} h_{\sigma}^{\nu} \partial_{\mu} \phi^{\dagger} \partial_{\nu} \phi+\frac{1}{16} h \square h \phi^{\dagger} \phi+\left(-\frac{\xi}{4}+\frac{1}{8}\right) \partial_{\mu} h \partial^{\mu} h \phi^{\dagger} \phi-2 \xi h^{\mu \nu} \partial_{\nu} \partial_{\lambda} h_{\mu}^{\lambda} \phi^{\dagger} \phi \\
& +\xi h^{\mu \nu} \square h_{\mu \nu} \phi^{\dagger} \phi-\xi \partial_{\nu} h^{\mu \nu} \partial_{\lambda} h_{\mu}^{\lambda} \phi^{\dagger} \phi+\frac{3}{4} \xi \partial_{\lambda} h_{\mu \nu} \partial^{\lambda} h^{\mu \nu} \phi^{\dagger} \phi-\frac{1}{2} \xi \partial_{\lambda} h^{\mu \nu} \partial_{\nu} h_{\mu}^{\lambda} \phi^{\dagger} \phi \\
& \left.\left(\xi-\frac{1}{4}\right) h^{\mu \nu} \partial_{\mu} \partial_{\nu} h \phi^{\dagger} \phi+\left(\xi-\frac{1}{4}\right) \partial_{\mu} h \partial_{\nu} h^{\mu \nu} \phi^{\dagger} \phi\right]
\end{aligned}
$$

The scalar-scalar-graviton vertex is:

$$
V_{s s h}^{\mu \mu}\left(p, p^{\prime}\right):-\frac{i}{4}\left(p^{\mu}+p^{\prime \mu}\right)^{2}-i\left(\xi-\frac{1}{4}\right)\left(\left(p^{\prime \mu}-p^{\mu}\right)^{2}-\eta^{\mu \mu}\left(p^{\prime}-p\right)^{2}\right)
$$

and there is a vertex with two scalars and two gravitons:

$$
\begin{aligned}
V_{s s h h}^{\mu \mu \nu \nu}\left(p, p^{\prime}, k, k^{\prime}\right): & i \eta^{\mu \nu}\left(p^{\prime \mu} p^{\nu}+p^{\mu} p^{\prime \nu}\right)-i\left[\left(\xi-\frac{1}{4}\right)\left(\eta^{\mu \mu} k^{\nu} k^{\nu}+\eta^{\nu \nu} k^{\mu} k^{\mu}\right)\right. \\
& \left.+2\left(\xi \eta^{\mu \nu} \eta^{\mu \nu}+\frac{1}{16} \eta^{\mu \mu} \eta^{\nu \nu}\right) k^{2}-4 \xi \eta^{\mu \nu} k^{\mu} k^{\nu}\right] \\
& -i\left[\left(\left(\frac{1}{4}-\frac{\xi}{2}\right) \eta^{\mu \mu} \eta^{\nu \nu}+\frac{3}{2} \xi \eta^{\mu \nu} \eta^{\mu \nu}\right) k \cdot k^{\prime}\right. \\
& \left.+\left(\xi-\frac{1}{4}\right)\left(\eta^{\mu \mu} k^{\nu} k^{\prime \nu}+\eta^{\nu \nu} k^{\mu} k^{\prime \mu}\right)-2 \xi \eta^{\mu \nu} k^{\mu} k^{\prime \nu}-\xi \eta^{\mu \nu} k^{\nu} k^{\prime \mu}\right]
\end{aligned}
$$

The result for the tadpole diagram is

$$
\tilde{\Theta}^{\mu \mu}=2^{-d-1} i \pi^{-d / 2} m^{d} \Gamma\left(-\frac{d}{2}\right) \eta^{\mu \mu}
$$

while the contribution from the seagull term is

$$
\begin{aligned}
\tilde{T}_{(s)}^{\mu \mu \nu \nu}(k)= & -2^{-4-d} i \pi^{-d / 2} m^{d-2} \Gamma\left(-\frac{d}{2}\right) \\
& \times\left(d k^{2}(1-4 \xi) \eta^{\mu \mu} \eta^{\nu \nu}+4 \eta^{\mu \nu} \eta^{\mu \nu}\left(4 m^{2}-d k^{2} \xi\right)+8 d \xi \eta^{\mu \nu} k^{\mu} k^{\nu}\right)
\end{aligned}
$$


Furthermore, the transverse part of the bubble diagram reads

$$
\begin{aligned}
\tilde{T}_{t}^{\mu \mu \nu \nu}(k)= & -\frac{1}{3 d\left(d^{2}-1\right) k^{4}} i 2^{-d-2} e^{-\frac{1}{2} i \pi d} \pi^{-d / 2}\left(-m^{2}\right)^{d / 2} m^{-2} \Gamma\left(1-\frac{d}{2}\right) \\
& {\left[\left(12\left(d^{2}-1\right) k^{4} m^{2}\left(8 \xi^{2}-8 \xi+1\right)+d\left(d^{2}-1\right) k^{6}\left(24 \xi^{2}-1\right)\right.\right.} \\
& +24 d k^{2} m^{4}(3-8 \xi)-192 k^{2} m^{4} \xi+96 m^{6} \\
& +\left(-6 k^{4} m^{2}\left(d^{2}(1-4 \xi)^{2}+d(8 \xi-2)-2\left(8 \xi^{2}-8 \xi+1\right)\right)\right. \\
& \left.\left.+24 k^{2} m^{4}(d(8 \xi-2)+8 \xi)-96 m^{6}\right){ }_{2} F_{1}\left[1,-\frac{d}{2} ;-\frac{1}{2} ; \frac{k^{2}}{4 m^{2}}\right]\right) \pi^{\mu \mu} \pi^{\nu \nu} \\
& +\left(-12 d^{2} k^{4} m^{2}+d\left(d^{2}-1\right) k^{6}+48 d k^{2} m^{4}-96 k^{2} m^{4}+12 k^{4} m^{2}+192 m^{6}\right. \\
& \left.\left.-12 m^{2}\left(k^{2}-4 m^{2}\right)^{2}{ }_{2} F_{1}\left[1,-\frac{d}{2} ;-\frac{1}{2} ; \frac{k^{2}}{4 m^{2}}\right]\right) \pi^{\mu \nu} \pi^{\mu \nu}\right]
\end{aligned}
$$

The expansion of the transverse part $\tilde{T}_{t}^{\mu \mu \nu \nu}(k)$ in the IR is

$$
\begin{aligned}
\tilde{T}_{t}^{\mu \mu \nu \nu}(k)= & 2^{-3-d} i m^{d-4} \pi^{-\frac{d}{2}} k^{4} \sum_{n=0}^{\infty} \frac{m^{-2 n} \Gamma\left(2+n-\frac{d}{2}\right)}{2^{n}(2 n+5) ! !} k^{2 n} \\
& \times\left(\pi^{\mu \nu} \pi^{\mu \nu}+\frac{a(n, \xi)}{2} \pi^{\mu \mu} \pi^{\nu \nu}\right)
\end{aligned}
$$

where $a(n, \xi)$ is a constant

$$
a(n, \xi)=(2 n+5)(2 n+3)(4 \xi-1)^{2}+2(2 n+5)(4 \xi-1)+1
$$

The non-transverse part of the bubble diagram is

$$
\begin{aligned}
\tilde{T}_{n t}^{\mu \mu \nu \nu}(k)= & \frac{2^{-4-d}}{3} i \pi^{-d / 2} m^{d-2} \Gamma\left(-\frac{d}{2}\right) \\
& \left(\eta^{\mu \nu} \eta^{\mu \nu}\left(24 m^{2}-2 d k^{2}\right)+4 d \eta^{\mu \nu} k^{\mu} k^{\nu}+2 d(6 \xi-1) \eta^{\mu \mu} k^{\nu} k^{\nu}\right. \\
& \left.+\eta^{\nu \nu}\left(\eta^{\mu \mu}\left(d k^{2}(5-24 \xi)+12 m^{2}\right)+2 d(6 \xi-1) k^{\mu} k^{\mu}\right)\right)
\end{aligned}
$$

The seagull diagram and the non-transverse part of 2-pt function together give

$$
\begin{aligned}
\tilde{T}_{(s)}^{\mu \mu \nu \nu}(k)+\tilde{T}_{n t}^{\mu \mu \nu \nu}(k)= & -2^{-d-2} i \pi^{-d / 2} m^{d} \Gamma\left(-\frac{d}{2}\right)\left(2 \eta^{\mu \nu} \eta^{\mu \nu}-\eta^{\mu \mu} \eta^{\nu \nu}\right) \\
& +2^{-d-1} i \pi^{-d / 2} m^{d-2}\left(\xi-\frac{1}{6}\right) \Gamma\left(1-\frac{d}{2}\right) k^{2}\left(\pi^{\mu \nu} \pi^{\mu \nu}-\pi^{\mu \mu} \pi^{\nu \nu}\right)
\end{aligned}
$$

Taking formulas (3.66), (3.67), (3.68) and (3.71) and substituting them in (3.12) we can see that the Ward identity is satisfied for any dimension $d$.

The one-loop 1-point correlator

$$
\begin{aligned}
\left\langle\left\langle T^{\mu \mu}(x)\right\rangle\right\rangle= & -2^{-d} m^{d} \pi^{-\frac{d}{2}}\left[\Gamma\left(-\frac{d}{2}\right) g^{\mu \mu}-\frac{2 \Gamma\left(1-\frac{d}{2}\right)}{m^{2}}\left(\xi-\frac{1}{6}\right) G^{\mu \mu}\right. \\
& +\sum_{n=2}^{\infty} \frac{(-1)^{n} m^{-2 n} \Gamma\left(n-\frac{d}{2}\right)}{2^{n}(2 n+1) ! !} \square^{n-2} \\
& \left.\times\left(-2 \square G^{\mu \mu}+\left(1-\frac{a(n, \xi)}{2}\right)\left(\eta^{\mu \mu} \square-\partial^{\mu} \partial^{\mu}\right) R\right)\right]+O\left(h^{2}\right)
\end{aligned}
$$


satisfies (3.9). For the effective action in the IR we obtain

$$
\begin{aligned}
W[h] \stackrel{\mathrm{IR}}{=} & 2^{-d} m^{d} \pi^{-\frac{d}{2}} \int d^{d} x \sqrt{g}\left[\Gamma\left(-\frac{d}{2}\right)-\frac{\Gamma\left(1-\frac{d}{2}\right)}{2 m^{2}}\left(\xi-\frac{1}{6}\right) R\right. \\
& \left.+\frac{\Gamma\left(2-\frac{d}{2}\right)}{120 m^{4}}\left(R_{\mu \nu \lambda \rho} R^{\mu \nu \lambda \rho}+\frac{a(0, \xi)}{2} R^{2}\right)+\ldots\right]+O\left(h^{3}\right)
\end{aligned}
$$

For $\xi=\frac{1}{6}$ (the conformal value) the third term in the expansion is proportional to

$$
\propto m^{d-4} \int d^{d} x \sqrt{g}\left(R_{\mu \nu \lambda \rho} R^{\mu \nu \lambda \rho}-\frac{1}{3} R^{2}\right)
$$

We can use the Gauss-Bonnet theorem

$$
R_{\mu \nu \lambda \rho} R^{\mu \nu \lambda \rho}-4 R_{\mu \nu} R^{\mu \nu}+R^{2}=\text { total derivative }
$$

to write the divergent part of the effective action in $d=4$ as a Weyl square density

$$
W \stackrel{\mathrm{IR}}{=}-\frac{1}{16 \pi^{2} \varepsilon} \int d^{4} x \sqrt{g}\left(m^{4}+\frac{1}{30} \mathcal{W}^{2}\right)+O\left(h^{3}\right)
$$

In the massless case $\left(m^{0}\right.$ is the dominating term in the UV) we have

$$
\tilde{T}^{\mu \mu \nu \nu}(k) \stackrel{\mathrm{UV}}{=} \frac{2^{-1-2 d} \pi^{\frac{3}{2}-\frac{d}{2}}\left(k^{2}\right)^{\frac{d}{2}}}{\left(-1+e^{i \pi d}\right) \Gamma\left(\frac{d+3}{2}\right)}\left(\pi^{\mu \nu} \pi^{\mu \nu}+\frac{b(d, \xi)}{2} \pi^{\mu \mu} \pi^{\nu \nu}\right)
$$

where

$$
b(d, \xi)=\left(d^{2}-1\right)(4 \xi-1)^{2}+2(d+1)(4 \xi-1)+1
$$

The effective action in the UV now becomes

$$
W \stackrel{\mathrm{UV}}{=}(-1)^{\frac{d}{2}} \frac{2^{-2-2 d+\left\lfloor\frac{d}{2}\right\rfloor} \pi^{\frac{3}{2}-\frac{d}{2}}}{\left(-1+e^{i \pi d}\right) \Gamma\left(\frac{d+3}{2}\right)} \int \mathrm{d}^{d} x\left(R^{\mu \nu \lambda \rho} \square^{\frac{d}{2}-2} R_{\mu \nu \lambda \rho}+\frac{b(d, \xi)}{2} R \square^{\frac{d}{2}-2} R\right)
$$

After we use (3.76) and put $\xi=\frac{1}{6}$ in $4 \mathrm{~d}$ we will again get the Weyl square density

$$
W \stackrel{\mathrm{UV}}{=} \int \mathrm{d}^{d} x \mathcal{W}^{2}
$$

\section{Correlators}

This section of the paper is a systematic collection of results concerning all types of twopoint correlators, including the mixed ones, for symmetric currents of spin up to 5 and in dimension $3 \leq d \leq 6$. It also contains results concerning the correlators of currents of any spin and in any dimensions, in the case of massless models, for which it is possible to write down very compact formulas. 
Since the volume of these formulas is rather big it is presented in the supplementary material. A part of this material is nevertheless kept here in the main text: sections 4.1 and 4.2 contain some representative calculations for spin 3 , and spin 3 - spin 5 mixed amplitudes.

We start by introducing the necessary quantities. The two point amplitudes in question for fermion and of scalar currents for spins up to 5, are schematically denoted as follows:

$$
\tilde{T}_{\mu_{1} \ldots \mu_{s_{1}} \nu_{1} \ldots \nu_{s_{2}}}(k) \equiv\left\langle\tilde{J}_{\mu_{1} \ldots \mu_{s_{1}}}(-k) \tilde{J}_{\nu_{1} \ldots \nu_{s_{2}}}(k)\right\rangle
$$

Scalar and fermion currents are given by

$$
J_{\mu_{1} \ldots \mu_{s}}^{\mathrm{s}}=i^{s} \varphi^{\dagger}\left(\stackrel{\leftrightarrow}{\partial}_{\mu}\right)^{s} \varphi, \quad J_{\mu_{1} \ldots \mu_{s}}^{\mathrm{f}}=i^{s-1} \bar{\psi} \gamma_{\mu}\left(\stackrel{\leftrightarrow}{\partial}_{\mu}\right)^{s-1} \psi
$$

(For fermions in case $s=0$ we use $J_{s=0}^{\mathrm{f}}=\bar{\psi} \psi$.) These currents will be henceforth referred to as simple currents. In the fermionic case the two point correlator is

$$
\tilde{T}_{\mu_{1} \ldots \mu_{s_{1}} \nu_{1} \ldots \nu_{s_{2}}}^{\mathrm{f}}(k)=-\int \frac{d^{d} p}{(2 \pi)^{d}} \operatorname{Tr}\left(\frac{i}{\not p-m} \gamma_{\sigma} \frac{i}{\not p-\not k-m} \gamma_{\tau}\right) V_{\mu_{1} \ldots \mu_{s_{1}}}^{\sigma} V_{\nu_{1} \ldots \nu_{s_{2}}}^{\tau}
$$

whereas in the scalar case it is

$$
\tilde{T}_{\mu_{1} \ldots \mu_{s_{1}} \nu_{1} \ldots \nu_{s_{2}}}^{\mathrm{s}}(k)=\int \frac{d^{d} p}{(2 \pi)^{d}} \frac{1}{\left(p^{2}-m^{2}\right)\left((p-k)^{2}-m^{2}\right)} V_{\mu_{1} \ldots \mu_{s_{1}}} V_{\nu_{1} \ldots \nu_{s_{2}}}
$$

with the Feynman vertices for fermions and scalars respectively

$$
V_{\mu_{1} \ldots \mu_{s}}^{\sigma}=i \delta_{\mu}^{\sigma}\left(2 p_{\mu}-k_{\mu}\right)^{s-1}, \quad V_{\mu_{1} \ldots \mu_{s}}=i\left(2 p_{\mu}-k_{\mu}\right)^{s}
$$

To label the correlators we often suppress indices and add the number of space-time dimensions in the subscript on the left hand side. Additionally, when $s_{1}, s_{2} \neq 0$, we split the amplitudes in the transverse and the non-transverse part, so for the correlator of e.g. fermionic spin- $s_{1}$ and spin- $s_{2}$ currents in $d$ dimensions we write:

$$
\tilde{T}_{s_{1}, s_{2}, d}^{\mathrm{f}}=\tilde{T}_{s_{1}, s_{2}, d}^{\mathrm{f}, \mathrm{t}}+\tilde{T}_{s_{1}, s_{2}, d}^{\mathrm{f}, \mathrm{nt}}
$$

There is no preferred way to do the splitting in (4.6) because one can always add some transverse quantity to $\tilde{T}^{\mathrm{t}}$ and subtract the same quantity from $\tilde{T}^{\text {nt }}$. However, it always happens that the non-transverse part can be chosen to be a polynomial in $k$ and $m$ (i.e. local). Here, we always make this choice so that the non-transverse part is local. After this choice is made there is still some remaining freedom in the splitting into the transverse and the non-transverse part in (4.6), nevertheless the quantities we define below do not depend on this remaining freedom.

One such quantity is $\tilde{T}_{s_{1}, s_{2}, d}^{\mathrm{f}, \mathrm{UV}-\mathrm{IR}}$, the difference between the UV and the IR expansions in the shortly explained sense. Since, as explained above, the non-transverse part is always local the non-transverse parts of UV and IR are the same and therefore cancel so that only transverse parts remain in the expression for $\tilde{T}_{s_{1}, s_{2}, d}^{\mathrm{f}, \mathrm{UV}-\mathrm{IR}}$

$$
\tilde{T}_{s_{1}, s_{2}, d}^{\mathrm{f}, \mathrm{UV}-\mathrm{IR}}=\tilde{T}_{s_{1}, s_{2}, d}^{\mathrm{f}, \mathrm{UV}}-\tilde{T}_{(0) s_{1}, s_{2}, d}^{\mathrm{f}, \mathrm{IR}}
$$


where the UV and IR expansions are denoted by $\tilde{T}_{s_{1}, s_{2}, d}^{\mathrm{ft}, \mathrm{UV}}$ and $\tilde{T}_{s_{1}, s_{2}, d}^{\mathrm{f}, \mathrm{IR}}$ respectively, and $\tilde{T}_{(0) s_{1}, s_{2}, d}^{\text {fIR }}$ is the part of the IR expansion of order $\mathcal{O}\left(m^{n}\right)$ with $n \geq 0$.

Another such quantities are the divergences of the correlators:

$$
\begin{aligned}
& \left(k \cdot \tilde{T}_{s_{1}, s_{2}, d}^{\mathrm{f}}\right)_{\mu_{2} \ldots \mu_{s_{1}} \nu_{1} \ldots \nu_{s_{2}}}=k^{\mu_{1}}\left(\tilde{T}_{s_{1}, s_{2}, d}^{\mathrm{f}, \mathrm{nt}}\right)_{\mu_{1} \ldots \mu_{s_{1}} \nu_{1} \ldots \nu_{s_{2}}} \\
& \left(\tilde{T}_{s_{1}, s_{2}, d}^{\mathrm{f}} \cdot k\right)_{\mu_{1} \ldots \mu_{s_{1}} \nu_{2} \ldots \nu_{s_{2}}}=k^{\nu_{1}}\left(\tilde{T}_{s_{1}, s_{2}, d}^{\mathrm{f}, \mathrm{nt}}\right)_{\mu_{1} \ldots \mu_{s_{1}} \nu_{1} \ldots \nu_{s_{2}}}
\end{aligned}
$$

The definitions (4.6), (4.7), (4.8) are analogous in the scalar case.

Before listing the results for the massive models, it is worth to show some general formulas (for any spin and any dimension) that it was possible to obtain for the massless case. (We recall that the results for the massless cases correspond to the dominant term in the UV expansion of the massive case.) In addition some general formulas are easy to write in terms of particular linear combination of the previous currents which become traceless in the massless case. These "traceless" versions of the currents can be defined in the following way:

$$
\tilde{T}_{\mu_{1} \ldots \mu_{s}}^{\mathrm{st}}=\sum_{l=0}^{\left\lfloor\frac{s}{2}\right\rfloor} a_{s, l}^{\mathrm{s}}\left(\square \pi_{\mu \mu}\right)^{l} \tilde{T}_{\mu_{1} \ldots \mu_{s-2 l}}^{\mathrm{s}}, \quad \tilde{T}_{\mu_{1} \ldots \mu_{s}}^{\mathrm{ft}}=\sum_{l=0}^{\left\lfloor\frac{s-1}{2}\right\rfloor} a_{s, l}^{\mathrm{f}}\left(\square \pi_{\mu \mu}\right)^{l} \tilde{T}_{\mu_{1} \ldots \mu_{s-2 l}}^{\mathrm{f}}
$$

where

$$
a_{s, l}^{\mathrm{s}}=\frac{(-1)^{l} s ! \Gamma\left(s+\frac{d-3}{2}-l\right)}{2^{2 l} l !(s-2 l) ! \Gamma\left(s+\frac{d-3}{2}\right)}, \quad a_{s, l}^{\mathrm{f}}=\frac{(-1)^{l}(s-1) ! \Gamma\left(s+\frac{d-3}{2}-l\right)}{2^{2 l} l !(s-2 l-1) ! \Gamma\left(s+\frac{d-3}{2}\right)}
$$

It is easy to see that amplitudes for two general spins $s_{1}$ and $s_{2}$ for the "traceless" currents can be written as linear combinations of the amplitudes (4.4) and (4.5) of the "simple" currents $(4.2)$

$$
\begin{aligned}
\tilde{T}_{\mu_{1} \ldots \mu_{s_{1}} \nu_{1} \ldots \nu_{s_{2}}}^{\mathrm{st}} & =\sum_{l=0}^{\left\lfloor\frac{s_{1}}{2}\right\rfloor} \sum_{k=0}^{\left\lfloor\frac{s_{2}}{2}\right\rfloor} a_{s_{1}, l}^{\mathrm{s}} a_{s_{2}, k}^{\mathrm{s}}\left(k^{2} \eta_{\mu \mu}-k_{\mu}^{2}\right)^{l}\left(k^{2} \eta_{\nu \nu}-k_{\nu}^{2}\right)^{k} \tilde{T}_{\mu_{1} \ldots \mu_{s_{1}-2 l} \nu_{1} \ldots \nu_{s_{2}-2 k}}^{\mathrm{s}} \\
\tilde{T}_{\mu_{1} \ldots \mu_{s_{1}} \nu_{1} \ldots \nu_{s_{2}}}^{\mathrm{ft}} & =\sum_{l=0}^{\left\lfloor\frac{s_{1}-1}{2}\right\rfloor} \sum_{k=0}^{\left\lfloor\frac{s_{2}-1}{2}\right\rfloor} a_{s_{1}, l}^{\mathrm{f}} a_{s_{2}, k}^{\mathrm{f}}\left(k^{2} \eta_{\mu \mu}-k_{\mu}^{2}\right)^{l}\left(k^{2} \eta_{\nu \nu}-k_{\nu}^{2}\right)^{k} \tilde{T}_{\mu_{1} \ldots \mu_{s_{1}-2 l} \nu_{1} \ldots \nu_{s_{2}-2 k}}^{\mathrm{f}}
\end{aligned}
$$

The result for the traceless currents in the massless limit is

$$
\begin{aligned}
\tilde{T}_{\mu_{1} \ldots \mu_{s} \nu_{1} \ldots \nu_{s}}^{\mathrm{st}, \text { massless }} & =(-1)^{s} \frac{2^{4-2 d-s} \pi^{\frac{3}{2}-\frac{d}{2}} s !\left(k^{2}\right)^{\frac{d}{2}+s-2}}{\left(-1+e^{i \pi d}\right) \Gamma\left(\frac{d+2 s-1}{2}\right)} \sum_{l=0}^{\left\lfloor\frac{s}{2}\right\rfloor} a_{s, l}^{\mathrm{s}} \pi_{\mu \mu}^{l} \pi_{\nu \nu}^{l} \pi_{\mu \nu}^{s-2 l} \\
& =(-1)^{s} \frac{2^{4-2 d-s} \pi^{\frac{3}{2}-\frac{d}{2}} s !\left(k^{2}\right)^{\frac{d}{2}+s-2}}{\left(-1+e^{i \pi d}\right) \Gamma\left(\frac{d+2 s-1}{2}\right)} \pi_{\mu \nu}^{s} F_{1}\left(\frac{1-s}{2},-\frac{s}{2}, \frac{5-d-2 s}{2}, \frac{\pi_{\mu \mu} \pi_{\nu \nu}}{\pi_{\mu \nu}^{2}}\right)
\end{aligned}
$$

We note that for traceless currents mixed spin terms are zero i.e. the result vanishes for spin $s_{1} \neq s_{2}$. For simple currents this is not the case and the general expression for spin 
$s_{1} \times s_{2}, s_{2} \geqslant s_{1}$ is

$$
\begin{aligned}
\tilde{T}_{\mu_{1} \ldots \mu_{s_{1}} \nu_{1} \ldots \nu_{s_{2}}}^{\text {s,massless }}= & (-1)^{\frac{s_{1}+s_{2}}{2}} \frac{\left(2\left\lfloor\frac{s_{2}+1}{2}\right\rfloor-1\right) ! !\left(2\left\lfloor\frac{s_{2}+1}{2}\right\rfloor\right) ! ! 2^{4-2 d-\frac{s_{1}+s_{2}}{2}} \pi^{\frac{3}{2}-\frac{d}{2}}\left(k^{2}\right)^{\frac{d+s_{1}+s_{2}}{2}-2}}{\left(2\left\lfloor\frac{s_{2}}{2}\right\rfloor-2\left\lfloor\frac{s_{1}}{2}\right\rfloor\right) ! !\left(-1+e^{i \pi d}\right) \Gamma\left(\frac{d+s_{1}+s_{2}-1}{2}\right)} \\
& \times \pi_{\nu \nu}^{\frac{s_{2}-s_{1}}{2}} \sum_{l=0}^{\left\lfloor\frac{s_{1}}{2}\right\rfloor} \frac{s_{1} !\left(s_{2}-s_{1}\right) ! !}{2^{\frac{l(l+1)}{2}}\left(s_{1}-2 l\right) !\left(s_{2}-s_{1}+2 l\right) ! !} \pi_{\mu \mu}^{l} \pi_{\nu \nu}^{l} \pi_{\mu \nu}^{s_{1}-2 l}
\end{aligned}
$$

For fermions in the massless limit it also happens that only the diagonal $\left(s_{1}=s_{2} \equiv s\right.$ and $s>0$ ) amplitudes survive for the traceless currents

$$
\begin{aligned}
\tilde{T}_{\mu_{1} \ldots \mu_{s} \nu_{1} \ldots \nu_{s}}^{\mathrm{ft}, \text { massless,even }}= & (-1)^{s} \frac{2^{3-2 d-s+\left\lfloor\frac{d}{2}\right\rfloor} \pi^{\frac{3}{2}-\frac{d}{2}}(s-1) !(d-3+s)\left(k^{2}\right)^{\frac{d}{2}+s-2}}{\left(-1+e^{i \pi d}\right) \Gamma\left(\frac{d+2 s-1}{2}\right)} \sum_{l=0}^{\left\lfloor\frac{s}{2}\right.} a_{s, l}^{\mathrm{s}} \pi_{\mu \mu}^{l} \pi_{\nu \nu}^{l} \pi_{\mu \nu}^{s-2 l} \\
= & (-1)^{s} \frac{2^{3-2 d-s+\left\lfloor\frac{d}{2}\right\rfloor} \pi^{\frac{3}{2}-\frac{d}{2}}(s-1) !(d-3+s)\left(k^{2}\right)^{\frac{d}{2}+s-2}}{\left(-1+e^{i \pi d}\right) \Gamma\left(\frac{d+2 s-1}{2}\right)} \\
& \times \pi_{\mu \nu}^{s}{ }_{2} F_{1}\left(\frac{1-s}{2},-\frac{s}{2}, \frac{5-d-2 s}{2}, \frac{\pi_{\mu \mu} \pi_{\nu \nu}}{\pi_{\mu \nu}^{2}}\right)
\end{aligned}
$$

The formula above is valid for $d \geq 4$ and for the even part in $d=3$. For the odd part in $d=3$ we obtain for traceless currents, for the dominant term in the UV, a general expression for spin $s_{1} \times s_{2}, s_{2} \geqslant s_{1}, s_{1}>0, s_{2}>0$

$$
\begin{aligned}
\tilde{T}_{\mu_{1} \ldots \mu_{s_{1}} \nu_{1} \ldots \nu_{2} ;}^{\mathrm{ft}, \mathrm{UV} \text { do dominant,odd }}= & (-1)^{\frac{s_{1}+s_{2}}{2}} \frac{i m k^{s_{1}+s_{2}-3}}{2^{s_{2}+1}} \pi_{\nu \nu}^{\frac{s_{2}-s_{1}}{\nu}} \sum_{l=0}^{\left\lfloor\frac{s_{1}-1}{2}\right\rfloor} \frac{(-1)^{l} \Gamma\left(s_{1}-l\right)}{2^{2 l} l ! \Gamma\left(s_{1}-2 l\right)} \pi_{\mu \mu}^{l} \pi_{\nu \nu}^{l} \pi_{\mu \nu}^{s_{1}-2 l-1} \epsilon_{\sigma \mu \nu} k^{\sigma} \\
= & (-1)^{\frac{s_{1}+s_{2}}{2}} \frac{i m k^{s_{1}+s_{2}-3}}{2^{s_{2}+1}} \pi_{\nu \nu}^{\frac{s_{2}-s_{1}}{2}} \pi_{\mu \nu}^{s_{1}-1} \\
& \times_{2} F_{1}\left(\frac{1-s_{1}}{2}, 1-\frac{s_{1}}{2}, 1-s_{1}, \frac{\pi_{\mu \mu} \pi_{\nu \nu}}{\pi_{\mu \nu}^{2}}\right) \epsilon_{\sigma \mu \nu} k^{\sigma}
\end{aligned}
$$

In appendix B we show that this formula is a straightforward generalization of the linearized action proposed long ago by Pope and Townsend, [8], for conformal higher spin fields. In the case of simple currents we instead get

$$
\begin{aligned}
\tilde{T}_{\mu_{1} \ldots \mu_{s_{1}} \nu_{1} \ldots \nu_{s_{2}} ; 3 D}^{\mathrm{f}, \mathrm{UV} \text { ominat,odd }}= & (-1)^{\frac{s_{1}+s_{2}}{2}} \frac{\left(2\left\lfloor\frac{s_{2}-1}{2}\right\rfloor\right) ! !\left(s_{1}+s_{2}-2\left\lfloor\frac{s_{1}-1}{2}\right\rfloor-3\right) ! ! m k^{s_{1}+s_{2}-3}}{2^{2}\left(s_{1}+s_{2}-2\right) ! !\left(2\left\lfloor\frac{s_{2}}{2}\right\rfloor-2\left\lfloor\frac{s_{1}}{2}\right\rfloor\right) ! !} \\
& \times \pi_{\nu \nu^{2}}^{\frac{s_{2}-s_{1}}{2}} \epsilon_{\sigma \mu \nu} k^{\sigma} \sum_{l=0}^{\left\lfloor\frac{s_{1}-1}{2}\right\rfloor} \frac{\left(s_{1}-1\right) !\left(s_{2}-s_{1}\right) ! !}{2^{\frac{l(l+1)}{2}}\left(s_{1}-2 l-1\right) !\left(s_{2}-s_{1}+2 l\right) ! !} \pi_{\mu \mu}^{l} \pi_{\nu \nu}^{l} \pi_{\mu \nu}^{s_{1}-2 l-1}
\end{aligned}
$$

In the case of simple currents it is possible to write the formula for the IR expansion of the transverse part:

$$
\begin{aligned}
\tilde{T}_{\mu_{1} \ldots \mu_{s_{1}} \nu_{1} \ldots \nu_{s_{2}}}^{\mathrm{f}, \mathrm{t}, \mathrm{IR}, \mathrm{d} D}= & (-1)^{\frac{s_{1}+s_{2}}{2}-1} \frac{\left(2\left\lfloor\frac{s_{2}-1}{2}\right\rfloor\right) ! !\left(s_{1}+s_{2}-2\left\lfloor\frac{s_{1}-1}{2}\right\rfloor-3\right) ! ! k^{s_{1}+s_{2}-2}}{2^{2} \pi\left(s_{1}+s_{2}-1\right) ! !\left(2\left\lfloor\frac{s_{2}}{2}\right\rfloor-2\left\lfloor\frac{s_{1}}{2}\right\rfloor\right) ! !} \\
& \times \pi_{\nu \nu}^{\frac{s_{2}-s_{1}}{2}} \epsilon_{\sigma \mu \nu} k^{\sigma} \sum_{l=0}^{\left\lfloor\frac{s_{1}-1}{2}\right\rfloor} \frac{\left(s_{1}-1\right) !\left(s_{2}-s_{1}\right) ! !}{2^{\frac{l(l+1)}{2}}\left(s_{1}-2 l-1\right) !\left(s_{2}-s_{1}+2 l\right) ! !} \pi_{\mu \mu}^{l} \pi_{\nu \nu}^{l} \pi_{\mu \nu}^{s_{1}-2 l-1}
\end{aligned}
$$


In the rest of the section we list the results for the massive case. The results are given for $d=3,4,5,6$ and spin $s \leq 5$. For even $d$, we use $d \rightarrow d+\varepsilon$ and expand around $\varepsilon$. For odd $d$ this is not necessary. It is convenient to use the following shorthand notation

$$
L_{n}=\frac{2}{\varepsilon}+\log \left(\frac{m^{2}}{4 \pi}\right)+\gamma-\sum_{k=1}^{n} \frac{1}{k}
$$

as well as

$$
\begin{aligned}
& K=\log \left(-\frac{k^{2}}{m^{2}}\right) \\
& P=\frac{2}{\varepsilon}+\log \left(-\frac{k^{2}}{4 \pi}\right)+\gamma
\end{aligned}
$$

We see that there is a relationship

$$
P=K+L_{0}
$$

Furthermore we define

$$
\begin{aligned}
& T=-\frac{2 i \operatorname{coth}^{-1}\left(\frac{2 m}{k}\right)}{\pi} \\
& S=\sqrt{4 m^{2}-k^{2}} \csc ^{-1}\left(\frac{2 m}{k}\right)
\end{aligned}
$$

It turns out that $T$ is useful in even dimensions $d$ and $S$ is useful in odd. The branches of the functions $T$ and $S$ are chosen such that the IR and UV expansions are

$$
\begin{aligned}
& T \stackrel{\text { IR }}{=}-\frac{i k}{\pi m}-\frac{i k^{3}}{12 \pi m^{3}}-\frac{i k^{5}}{80 \pi m^{5}}+\ldots \\
& S \stackrel{\text { IR }}{=} k-\frac{k^{3}}{12 m^{2}}-\frac{k^{5}}{120 m^{4}}+\ldots
\end{aligned}
$$

and

$$
\begin{aligned}
T & \stackrel{\mathrm{UV}}{=} 1-\frac{4 i m}{\pi k}-\frac{16 i m^{3}}{3 \pi k^{3}}-\frac{64 i m^{5}}{5 \pi k^{5}}+\ldots \\
S & \stackrel{\mathrm{UV}}{=} \frac{k K}{2}-\frac{m^{2}(1+K)}{k}+\frac{m^{4}(1-2 K)}{2 k^{3}}+\frac{m^{6}(5-6 K)}{3 k^{5}}+\ldots
\end{aligned}
$$

In the results for UV-IR which follow, the difference is shown for the terms containing the powers of $m$ and $k$ that "overlap" in UV and IR in sense that those powers appear both in UV and in IR expansions. The rest, i.e. the UV expansion that does not overlap with the IR, is denoted by ellipses.

In the following two sections we list the results for fermions for spin 3 and mixed spin 3 -spin 5 amplitudes for dimensions 3 and 4 . Section 4.1 contains the full transverse analytic expressions of the correlators. Section 4.2 contains the UV and IR expansions of the latter, as well as the above-mentioned UV-IR expressions.

The method to obtain the results below has been explained in [1] and is largely based on the approach of Davydychev and collaborators, [62-64], see [65]. 


\subsection{Fermion amplitudes for spins $3 \times 3$ and $3 \times 5$}

Fermions, spin $3 \times 3$, dimension 3 :

$$
\begin{aligned}
\tilde{T}_{3,3 ; 3 \mathrm{D}}^{\mathrm{f}, \mathrm{t}}= & k^{6} \pi_{\mu \nu}^{3}\left(\frac{i}{2 \pi}\left(\frac{1}{8} \frac{m}{k^{2}}-\frac{1}{3} \frac{m^{3}}{k^{4}}+2 \frac{m^{5}}{k^{6}}\right)+T\left(\frac{1}{64} \frac{1}{k}-\frac{1}{16} \frac{m^{2}}{k^{3}}-\frac{1}{4} \frac{m^{4}}{k^{5}}+\frac{m^{6}}{k^{7}}\right)\right)+ \\
& +k^{6} \pi_{\mu \mu} \pi_{\mu \nu} \pi_{\nu \nu}\left(\frac{i}{2 \pi}\left(-\frac{1}{16} \frac{m}{k^{2}}-\frac{3}{2} \frac{m^{3}}{k^{4}}+3 \frac{m^{5}}{k^{6}}\right)+\right. \\
& \left.+T\left(-\frac{1}{128} \frac{1}{k}+\frac{5}{32} \frac{m^{2}}{k^{3}}-\frac{7}{8} \frac{m^{4}}{k^{5}}+\frac{3}{2} \frac{m^{6}}{k^{7}}\right)\right)+ \\
& +k^{4}(k \cdot \epsilon)_{\mu \nu} \pi_{\mu \nu}^{2}\left(\frac{1}{4 \pi}\left(-3 \frac{m^{2}}{k^{2}}+4 \frac{m^{4}}{k^{4}}\right)+i T\left(-\frac{1}{16} \frac{m}{k}+\frac{1}{2} \frac{m^{3}}{k^{3}}-\frac{m^{5}}{k^{5}}\right)\right)+ \\
& +k^{4}(k \cdot \epsilon)_{\mu \nu} \pi_{\mu \mu} \pi_{\nu \nu}\left(\frac{1}{2 \pi}\left(\frac{1}{4} \frac{m^{2}}{k^{2}}+\frac{m^{4}}{k^{4}}\right)+i T\left(-\frac{1}{32} \frac{m}{k}+\frac{1}{4} \frac{m^{3}}{k^{3}}-\frac{1}{2} \frac{m^{5}}{k^{5}}\right)\right) \\
\tilde{T}_{3,3 ; 3 \mathrm{D}}^{\mathrm{f,nt}}= & \left(k_{\nu}^{2} \eta_{\mu \mu} \eta_{\mu \nu}+k_{\mu} k_{\nu} \eta_{\mu \mu} \eta_{\nu \nu}+k_{\mu}^{2} \eta_{\mu \nu} \eta_{\nu \nu}\right)\left(-\frac{4 i}{3 \pi} m^{3}\right)+ \\
& +\eta_{\mu \mu} \eta_{\mu \nu} \eta_{\nu \nu}\left(\frac{i}{3 \pi}\left(4 k^{2} m^{3}-\frac{64}{5} m^{5}\right)\right)+\eta_{\mu \nu}^{3}\left(-\frac{32 i}{15 \pi} m^{5}\right)+ \\
& +k_{\mu} k_{\nu}(k \cdot \epsilon)_{\mu \nu} \eta_{\mu \nu}\left(-\frac{2}{\pi} m^{2}\right)+(k \cdot \epsilon)_{\mu \nu} \eta_{\mu \mu} \eta_{\nu \nu}\left(-\frac{4}{3 \pi} m^{4}\right)+ \\
& +(k \cdot \epsilon)_{\mu \nu} \eta_{\mu \nu}^{2}\left(\frac{1}{3 \pi}\left(3 k^{2} m^{2}-8 m^{4}\right)\right)
\end{aligned}
$$

Fermions, spin $3 \times 3$, dimension 4 :

$$
\begin{aligned}
\tilde{T}_{3,3 ; 4 \mathrm{D}}^{\mathrm{ft}}= & k^{6} \pi_{\mu \nu}^{3}\left(\frac{i}{5 \pi^{2}}\left(\left(-\frac{599}{4410}+\frac{L_{0}}{21}\right)+\left(\frac{247}{315}-\frac{L_{0}}{3}\right) \frac{m^{2}}{k^{2}}+\frac{8}{7} \frac{m^{4}}{k^{4}}-\frac{32}{7} \frac{m^{6}}{k^{6}}\right)+\right. \\
& \left.+\frac{i S}{7 \pi^{2}}\left(\frac{2}{15} \frac{1}{k}-\frac{2}{3} \frac{m^{2}}{k^{3}}-\frac{16}{15} \frac{m^{4}}{k^{5}}+\frac{32}{5} \frac{m^{6}}{k^{7}}\right)\right)+ \\
& +k^{6} \pi_{\mu \mu} \pi_{\mu \nu} \pi_{\nu \nu}\left(\frac { i } { 5 \pi ^ { 2 } } \left(\left(\frac{457}{8820}-\frac{L_{0}}{84}\right)+\left(-\frac{382}{315}+\frac{L_{0}}{3}\right) \frac{m^{2}}{k^{2}}+\frac{92}{21} \frac{m^{4}}{k^{4}}-\right.\right. \\
& \left.\left.-\frac{48}{7} \frac{m^{6}}{k^{6}}\right)+\frac{i S}{7 \pi^{2}}\left(-\frac{1}{30} \frac{1}{k}+\frac{13}{15} \frac{m^{2}}{k^{3}}-\frac{16}{3} \frac{m^{4}}{k^{5}}+\frac{48}{5} \frac{m^{6}}{k^{7}}\right)\right) \\
\tilde{T}_{3,3 ; 4 \mathrm{D}}^{\mathrm{f}, \mathrm{nt}}= & \left(k_{\nu}^{2} \eta_{\mu \mu} \eta_{\mu \nu}+k_{\mu} k_{\nu} \eta_{\mu \mu} \eta_{\nu \nu}+k_{\mu}^{2} \eta_{\mu \nu} \eta_{\nu \nu}\right)\left(\frac{i L_{2}}{2 \pi^{2}} m^{4}\right)+ \\
& +\eta_{\mu \mu} \eta_{\mu \nu} \eta_{\nu \nu}\left(\frac{i}{\pi^{2}}\left(-\frac{L_{2}}{2} k^{2} m^{4}+\frac{4 L_{3}}{3} m^{6}\right)\right)+\eta_{\mu \nu}^{3}\left(\frac{2 i L_{3}}{3 \pi^{2}} m^{6}\right)
\end{aligned}
$$


Fermions, spin $3 \times 5$, dimension 3 :

$$
\begin{aligned}
& \tilde{T}_{3,5 ; 3 \mathrm{D}}^{\mathrm{f}, \mathrm{t}}=k^{8} \pi_{\mu \nu}^{3} \pi_{\nu \nu}\left(\frac{i}{4 \pi}\left(-\frac{3}{16} \frac{m}{k^{2}}+\frac{3}{4} \frac{m^{3}}{k^{4}}-\frac{31}{3} \frac{m^{5}}{k^{6}}+20 \frac{m^{7}}{k^{8}}\right)+\right. \\
& \left.+T\left(-\frac{3}{256} \frac{1}{k}+\frac{1}{16} \frac{m^{2}}{k^{3}}+\frac{3}{8} \frac{m^{4}}{k^{5}}-3 \frac{m^{6}}{k^{7}}+5 \frac{m^{8}}{k^{9}}\right)\right)+ \\
& +k^{8} \pi_{\mu \mu} \pi_{\mu \nu} \pi_{\nu \nu}^{2}\left(\frac{i}{4 \pi}\left(\frac{7}{64} \frac{m}{k^{2}}+\frac{155}{48} \frac{m^{3}}{k^{4}}-\frac{47}{4} \frac{m^{5}}{k^{6}}+15 \frac{m^{7}}{k^{8}}\right)+\right. \\
& \left.+T\left(\frac{7}{1024} \frac{1}{k}-\frac{9}{64} \frac{m^{2}}{k^{3}}+\frac{33}{32} \frac{m^{4}}{k^{5}}-\frac{13}{4} \frac{m^{6}}{k^{7}}+\frac{15}{4} \frac{m^{8}}{k^{9}}\right)\right)+ \\
& +k^{6}(k \cdot \epsilon)_{\mu \nu} \pi_{\mu \nu}^{2} \pi_{\nu \nu}\left(\frac{1}{\pi}\left(\frac{3}{4} \frac{m^{2}}{k^{2}}-\frac{8}{3} \frac{m^{4}}{k^{4}}+4 \frac{m^{6}}{k^{6}}\right)+\right. \\
& \left.+i T\left(\frac{1}{16} \frac{m}{k}-\frac{3}{4} \frac{m^{3}}{k^{3}}+3 \frac{m^{5}}{k^{5}}-4 \frac{m^{7}}{k^{7}}\right)\right)+ \\
& +k^{6}(k \cdot \epsilon)_{\mu \nu} \pi_{\mu \mu} \pi_{\nu \nu}^{2}\left(\frac{1}{\pi}\left(-\frac{1}{16} \frac{m^{2}}{k^{2}}-\frac{2}{3} \frac{m^{4}}{k^{4}}+\frac{m^{6}}{k^{6}}\right)+\right. \\
& \left.+i T\left(\frac{1}{64} \frac{m}{k}-\frac{3}{16} \frac{m^{3}}{k^{3}}+\frac{3}{4} \frac{m^{5}}{k^{5}}-\frac{m^{7}}{k^{7}}\right)\right) \\
& \tilde{T}_{3,5 ; 3 \mathrm{D}}^{\mathrm{f}, \mathrm{nt}}=\left(k_{\mu} k_{\nu}^{3} \eta_{\mu \mu} \eta_{\nu \nu}+k_{\mu}^{2} k_{\nu}^{2} \eta_{\mu \nu} \eta_{\nu \nu}\right)\left(-\frac{8 i}{3 \pi} m^{3}\right)+\left(k_{\nu}^{4} \eta_{\mu \mu} \eta_{\mu \nu}+k_{\mu}^{3} k_{\nu} \eta_{\nu \nu}^{2}\right)\left(-\frac{4 i}{3 \pi} m^{3}\right)+ \\
& +\left(k_{\mu} k_{\nu} \eta_{\mu \mu} \eta_{\nu \nu}^{2}+k_{\mu}^{2} \eta_{\mu \nu} \eta_{\nu \nu}^{2}\right)\left(\frac{i}{\pi}\left(\frac{4}{3} k^{2} m^{3}-\frac{32}{5} m^{5}\right)\right)+ \\
& +k_{\nu}^{2} \eta_{\mu \mu} \eta_{\mu \nu} \eta_{\nu \nu}\left(\frac{i}{\pi}\left(\frac{8}{3} k^{2} m^{3}-\frac{64}{5} m^{5}\right)\right)+k_{\mu} k_{\nu} \eta_{\mu \nu}^{2} \eta_{\nu \nu}\left(-\frac{64 i}{5 \pi} m^{5}\right)+ \\
& +k_{\nu}^{2} \eta_{\mu \nu}^{3}\left(-\frac{64 i}{15 \pi} m^{5}\right)+\eta_{\mu \mu} \eta_{\mu \nu} \eta_{\nu \nu}^{2}\left(\frac{i}{\pi}\left(-\frac{4}{3} k^{4} m^{3}+\frac{32}{5} k^{2} m^{5}-\frac{64}{5} m^{7}\right)\right)+ \\
& +\eta_{\mu \nu}^{3} \eta_{\nu \nu}\left(\frac{i}{5 \pi}\left(\frac{64}{3} k^{2} m^{5}-\frac{512}{7} m^{7}\right)\right)+k_{\mu}^{2} k_{\nu}^{2}(k \cdot \epsilon)_{\mu \nu} \eta_{\nu \nu}\left(-\frac{1}{\pi} m^{2}\right)+ \\
& +k_{\mu} k_{\nu}^{3}(k \cdot \epsilon)_{\mu \nu} \eta_{\mu \nu}\left(-\frac{2}{\pi} m^{2}\right)+k_{\nu}^{2}(k \cdot \epsilon)_{\mu \nu} \eta_{\mu \mu} \eta_{\nu \nu}\left(-\frac{8}{3 \pi} m^{4}\right)+ \\
& +k_{\mu}^{2}(k \cdot \epsilon)_{\mu \nu} \eta_{\nu \nu}^{2}\left(-\frac{4}{3 \pi} m^{4}\right)+k_{\mu} k_{\nu}(k \cdot \epsilon)_{\mu \nu} \eta_{\mu \nu} \eta_{\nu \nu}\left(\frac{1}{3 \pi}\left(6 k^{2} m^{2}-32 m^{4}\right)\right)+ \\
& +k_{\nu}^{2}(k \cdot \epsilon)_{\mu \nu} \eta_{\mu \nu}^{2}\left(\frac{1}{3 \pi}\left(3 k^{2} m^{2}-16 m^{4}\right)\right)+ \\
& +(k \cdot \epsilon)_{\mu \nu} \eta_{\mu \mu} \eta_{\nu \nu}^{2}\left(\frac{1}{\pi}\left(\frac{4}{3} k^{2} m^{4}-\frac{16}{5} m^{6}\right)\right)+ \\
& +(k \cdot \epsilon)_{\mu \nu} \eta_{\mu \nu}^{2} \eta_{\nu \nu}\left(\frac{1}{\pi}\left(-k^{4} m^{2}+\frac{16}{3} k^{2} m^{4}-\frac{64}{5} m^{6}\right)\right)
\end{aligned}
$$


Fermions, spin $3 \times 5$, dimension 4 :

$$
\begin{aligned}
\tilde{T}_{3,5 ; 4 \mathrm{D}}^{\mathrm{f,t}}= & k^{8} \pi_{\mu \nu}^{3} \pi_{\nu \nu}\left(\frac { i } { 7 \pi ^ { 2 } } \left(\left(\frac{1937}{14175}-\frac{2 L_{0}}{45}\right)+\left(-\frac{1622}{1575}+\frac{2 L_{0}}{5}\right) \frac{m^{2}}{k^{2}}-\frac{32}{15} \frac{m^{4}}{k^{4}}+\right.\right. \\
& \left.+\frac{2432}{135} \frac{m^{6}}{k^{6}}-\frac{256}{9} \frac{m^{8}}{k^{8}}\right)+ \\
& \left.+\frac{i S}{3 \pi^{2}}\left(-\frac{4}{105} \frac{1}{k}+\frac{4}{15} \frac{m^{2}}{k^{3}}+\frac{16}{35} \frac{m^{4}}{k^{5}}-\frac{704}{105} \frac{m^{6}}{k^{7}}+\frac{256}{21} \frac{m^{8}}{k^{9}}\right)\right)+ \\
& +k^{8} \pi_{\mu \mu} \pi_{\mu \nu} \pi_{\nu \nu}^{2}\left(\frac { i } { \pi ^ { 2 } } \left(\left(-\frac{1231}{132300}+\frac{L_{0}}{420}\right)+\left(\frac{258}{1225}-\frac{2 L_{0}}{35}\right) \frac{m^{2}}{k^{2}}-\frac{104}{105} \frac{m^{4}}{k^{4}}+\right.\right. \\
& \left.+\frac{128}{45} \frac{m^{6}}{k^{6}}-\frac{64}{21} \frac{m^{8}}{k^{8}}\right)+ \\
& \left.+\frac{i S}{\pi^{2}}\left(\frac{1}{210} \frac{1}{k}-\frac{11}{105} \frac{m^{2}}{k^{3}}+\frac{4}{5} \frac{m^{4}}{k^{5}}-\frac{272}{105} \frac{m^{6}}{k^{7}}+\frac{64}{21} \frac{m^{8}}{k^{9}}\right)\right) \\
\tilde{T}_{3,5 ; 4 \mathrm{D}}^{\mathrm{f,nt}}= & \left(k_{\mu} k_{\nu}^{3} \eta_{\mu \mu} \eta_{\nu \nu}+k_{\mu}^{2} k_{\nu}^{2} \eta_{\mu \nu} \eta_{\nu \nu}\right)\left(\frac{i L_{2}}{\pi^{2}} m^{4}\right)+\left(k_{\nu}^{4} \eta_{\mu \mu} \eta_{\mu \nu}+k_{\mu}^{3} k_{\nu} \eta_{\nu \nu}^{2}\right)\left(\frac{i L_{2}}{2 \pi^{2}} m^{4}\right)+ \\
& +\left(k_{\mu} k_{\nu} \eta_{\mu \mu} \eta_{\nu \nu}^{2}+k_{\mu}^{2} \eta_{\mu \nu} \eta_{\nu \nu}^{2}\right)\left(\frac{i}{2 \pi^{2}}\left(-L_{2} k^{2} m^{4}+4 L_{3} m^{6}\right)\right)+ \\
& +k_{\nu}^{2} \eta_{\mu \mu} \eta_{\mu \nu} \eta_{\nu \nu}\left(\frac{i}{\pi^{2}}\left(-L_{2} k^{2} m^{4}+4 L_{3} m^{6}\right)\right)+k_{\mu} k_{\nu} \eta_{\mu \nu}^{2} \eta_{\nu \nu}\left(\frac{4 i L_{3}}{\pi^{2}} m^{6}\right)+ \\
& +k_{\nu}^{2} \eta_{\mu \nu}^{3}\left(\frac{4 i L_{3}}{3 \pi^{2}} m^{6}\right)+\eta_{\mu \mu} \eta_{\mu \nu} \eta_{\nu \nu}^{2}\left(\frac{i}{2 \pi^{2}}\left(L_{2} k^{4} m^{4}-4 L_{3} k^{2} m^{6}+7 L_{4} m^{8}\right)\right)+ \\
& +\eta_{\mu \nu}^{3} \eta_{\nu \nu}\left(\frac{i}{3 \pi^{2}}\left(-4 L_{3} k^{2} m^{6}+12 L_{4} m^{8}\right)\right)
\end{aligned}
$$

\subsection{Expansions in UV and IR for fermions for spins $3 \times 3$ and $3 \times 5$}

Fermions, spin $3 \times 3$, dimension 3 :

$$
\begin{aligned}
\tilde{T}_{3,3 ; 3 \mathrm{D}}^{\mathrm{f}, \mathrm{UV}}= & k^{6} \pi_{\mu \nu}^{3}\left(\frac{1}{64} \frac{1}{k}-\frac{1}{16} \frac{m^{2}}{k^{3}}-\frac{1}{4} \frac{m^{4}}{k^{5}}+\frac{32 i}{15 \pi} \frac{m^{5}}{k^{6}}+\frac{m^{6}}{k^{7}}-\frac{256 i}{105 \pi} \frac{m^{7}}{k^{8}}-\frac{512 i}{315 \pi} \frac{m^{9}}{k^{10}}-\right. \\
& \left.-\frac{8192 i}{3465 \pi} \frac{m^{11}}{k^{12}}+\ldots\right)+ \\
& +k^{6} \pi_{\mu \mu} \pi_{\mu \nu} \pi_{\nu \nu}\left(-\frac{1}{128} \frac{1}{k}+\frac{5}{32} \frac{m^{2}}{k^{3}}-\frac{4 i}{3 \pi} \frac{m^{3}}{k^{4}}-\frac{7}{8} \frac{m^{4}}{k^{5}}+\frac{64 i}{15 \pi} \frac{m^{5}}{k^{6}}+\frac{3}{2} \frac{m^{6}}{k^{7}}-\right. \\
& \left.-\frac{64 i}{21 \pi} \frac{m^{7}}{k^{8}}-\frac{512 i}{315 \pi} \frac{m^{9}}{k^{10}}-\frac{1024 i}{495 \pi} \frac{m^{11}}{k^{12}}+\ldots\right)+ \\
& +k^{4}(k \cdot \epsilon)_{\mu \nu} \pi_{\mu \nu}^{2}\left(-\frac{i}{16} \frac{m}{k}-\frac{1}{\pi} \frac{m^{2}}{k^{2}}+\frac{i}{2} \frac{m^{3}}{k^{3}}+\frac{8}{3 \pi} \frac{m^{4}}{k^{4}}-i \frac{m^{5}}{k^{5}}-\frac{32}{15 \pi} \frac{m^{6}}{k^{6}}-\right. \\
& \left.-\frac{128}{105 \pi} \frac{m^{8}}{k^{8}}-\frac{512}{315 \pi} \frac{m^{10}}{k^{10}}-\frac{2048}{693 \pi} \frac{m^{12}}{k^{12}}+\ldots\right)+
\end{aligned}
$$




$$
\begin{aligned}
& +k^{4}(k \cdot \epsilon)_{\mu \nu} \pi_{\mu \mu} \pi_{\nu \nu}\left(-\frac{i}{32} \frac{m}{k}+\frac{i}{4} \frac{m^{3}}{k^{3}}+\frac{4}{3 \pi} \frac{m^{4}}{k^{4}}-\frac{i}{2} \frac{m^{5}}{k^{5}}-\frac{16}{15 \pi} \frac{m^{6}}{k^{6}}-\right. \\
& \left.-\frac{64}{105 \pi} \frac{m^{8}}{k^{8}}-\frac{256}{315 \pi} \frac{m^{10}}{k^{10}}-\frac{1024}{693 \pi} \frac{m^{12}}{k^{12}}+\ldots\right) \\
\tilde{T}_{3,3 ; 3 \mathrm{D}}^{\mathrm{ft,IR}}= & k^{6} \pi_{\mu \nu}^{3}\left(\frac { i } { 3 \pi } \left(\frac{2}{5} \frac{m}{k^{2}}-\frac{1}{35} \frac{1}{m}-\frac{1}{840} \frac{k^{2}}{m^{3}}-\frac{1}{9240} \frac{k^{4}}{m^{5}}-\frac{5}{384384} \frac{k^{6}}{m^{7}}-\frac{1}{549120} \frac{k^{8}}{m^{9}}-\right.\right. \\
& \left.\left.-\frac{7}{24893440} \frac{k^{10}}{m^{11}}+\ldots\right)\right)+ \\
& +k^{6} \pi_{\mu \mu} \pi_{\mu \nu} \pi_{\nu \nu}\left(\frac { i } { 3 \pi } \left(-\frac{2}{5} \frac{m}{k^{2}}+\frac{1}{140} \frac{1}{m}-\frac{1}{73920} \frac{k^{4}}{m^{5}}-\frac{1}{384384} \frac{k^{6}}{m^{7}}-\right.\right. \\
& \left.\left.-\frac{1}{2196480} \frac{k^{8}}{m^{9}}-\frac{1}{12446720} \frac{k^{10}}{m^{11}}+\ldots\right)\right)+ \\
& +k^{4}(k \cdot \epsilon)_{\mu \nu} \pi_{\mu \nu}^{2}\left(\frac { 1 } { 3 \pi } \left(-\frac{m^{2}}{k^{2}}-\frac{1}{10}-\frac{1}{280} \frac{k^{2}}{m^{2}}-\frac{1}{3360} \frac{k^{4}}{m^{4}}-\frac{1}{29568} \frac{k^{6}}{m^{6}}-\right.\right. \\
& \left.\left.-\frac{1}{219648} \frac{k^{8}}{m^{8}}-\frac{1}{1464320} \frac{k^{10}}{m^{10}}-\frac{1}{9052160} \frac{k^{12}}{m^{12}}+\ldots\right)\right)+ \\
& +k^{4}(k \cdot \epsilon)_{\mu \nu} \pi_{\mu \mu} \pi_{\nu \nu}\left(\frac { 1 } { 3 \pi } \left(\frac{m^{2}}{k^{2}}-\frac{1}{20}-\frac{1}{560} \frac{k^{2}}{m^{2}}-\frac{1}{6720} \frac{k^{4}}{m^{4}}-\frac{1}{59136} \frac{k^{6}}{m^{6}}-\right.\right. \\
& \left.\left.-\frac{1}{439296} \frac{k^{8}}{m^{8}}-\frac{1}{2928640} \frac{k^{10}}{m^{10}}-\frac{1}{18104320} \frac{k^{12}}{m^{12}}+\ldots\right)\right) \\
\tilde{T}_{3,3 ; 3 \mathrm{D}}^{\mathrm{f} \mathrm{UV}-\mathrm{IR}}= & k^{4}(k \cdot \epsilon)_{\mu \nu} \pi_{\mu \nu}^{2}\left(-\frac{2}{3 \pi} \frac{m^{2}}{k^{2}}\right)+\ldots
\end{aligned}
$$

Fermions, spin $3 \times 3$, dimension 4 :

$$
\begin{aligned}
\tilde{T}_{3,3 ; 4 \mathrm{D}}^{\mathrm{f}, \mathrm{UV}}= & k^{6} \pi_{\mu \nu}^{3}\left(\frac { i } { \pi ^ { 2 } } \left(\left(-\frac{599}{22050}+\frac{P}{105}\right)+\left(\frac{31}{225}-\frac{P}{15}\right) \frac{m^{2}}{k^{2}}+\frac{1}{3} \frac{m^{4}}{k^{4}}+\right.\right. \\
& +\left(-\frac{7}{9}+\frac{2 K}{3}\right) \frac{m^{6}}{k^{6}}-\left(\frac{19}{18}+\frac{2 K}{3}\right) \frac{m^{8}}{k^{8}}+\left(\frac{1}{50}-\frac{2 K}{5}\right) \frac{m^{10}}{k^{10}}+ \\
& \left.\left.+\left(\frac{56}{225}-\frac{8 K}{15}\right) \frac{m^{12}}{k^{12}}+\ldots\right)\right)+ \\
& +k^{6} \pi_{\mu \mu} \pi_{\mu \nu} \pi_{\nu \nu}\left(\frac { i } { \pi ^ { 2 } } \left(\left(\frac{457}{44100}-\frac{P}{420}\right)+\left(-\frac{107}{450}+\frac{P}{15}\right) \frac{m^{2}}{k^{2}}+\right.\right. \\
& +\left(\frac{3}{4}-\frac{K}{2}\right) \frac{m^{4}}{k^{4}}+\left(-\frac{5}{9}+\frac{4 K}{3}\right) \frac{m^{6}}{k^{6}}-\left(\frac{113}{72}+\frac{5 K}{6}\right) \frac{m^{8}}{k^{8}}- \\
& \left.\left.-\left(\frac{7}{150}+\frac{2 K}{5}\right) \frac{m^{10}}{k^{10}}+\left(\frac{151}{900}-\frac{7 K}{15}\right) \frac{m^{12}}{k^{12}}+\ldots\right)\right)
\end{aligned}
$$




$$
\begin{aligned}
\tilde{T}_{3,3 ; 4 \mathrm{D}}^{\mathrm{f}, \mathrm{t}, \mathrm{IR}}= & k^{6} \pi_{\mu \nu}^{3}\left(\frac { i } { 3 \pi ^ { 2 } } \left(\left(\frac{1}{5}-\frac{L_{0}}{5}\right) \frac{m^{2}}{k^{2}}+\frac{L_{0}}{35}-\frac{1}{420} \frac{k^{2}}{m^{2}}-\frac{1}{6930} \frac{k^{4}}{m^{4}}-\frac{1}{72072} \frac{k^{6}}{m^{6}}-\right.\right. \\
& \left.\left.-\frac{1}{600600} \frac{k^{8}}{m^{8}}-\frac{1}{4375800} \frac{k^{10}}{m^{10}}-\frac{1}{29099070} \frac{k^{12}}{m^{12}}+\ldots\right)\right)+ \\
& +k^{6} \pi_{\mu \mu} \pi_{\mu \nu} \pi_{\nu \nu}\left(\frac { i } { 3 \pi ^ { 2 } } \left(\left(-\frac{1}{5}+\frac{L_{0}}{5}\right) \frac{m^{2}}{k^{2}}-\frac{L_{0}}{140}-\frac{1}{55440} \frac{k^{4}}{m^{4}}-\frac{1}{360360} \frac{k^{6}}{m^{6}}-\right.\right. \\
& \left.\left.-\frac{1}{2402400} \frac{k^{8}}{m^{8}}-\frac{1}{15315300} \frac{k^{10}}{m^{10}}-\frac{1}{93117024} \frac{k^{12}}{m^{12}}+\ldots\right)\right) \\
\tilde{T}_{3,3 ; 4 \mathrm{D}}^{\mathrm{f}, \mathrm{UV}-\mathrm{IR}}= & k^{6} \pi_{\mu \nu}^{3}\left(\frac{i}{15 \pi^{2}}\left(\left(-\frac{599}{1470}+\frac{K}{7}\right)+\left(\frac{16}{15}-K\right) \frac{m^{2}}{k^{2}}\right)\right)+ \\
& +k^{6} \pi_{\mu \mu} \pi_{\mu \nu} \pi_{\nu \nu}\left(\frac{i}{15 \pi^{2}}\left(\left(\frac{457}{2940}-\frac{K}{28}\right)+\left(-\frac{77}{30}+K\right) \frac{m^{2}}{k^{2}}\right)\right)+\ldots
\end{aligned}
$$

\subsection{All types of correlators}

The contents of this section are presented in the supplementary material.

\section{Conclusion}

We finally sum up our main results. In this paper we have pursued further the program started in [1], considering in particular the quadratic part of the effective action.

First of all, we have discussed the relevant issue of the geometric interpretation of the obtained effective actions. The basic outcome of [1] was that, upon considering onshell conserved higher spin currents, the corresponding effective actions inherit an off-shell gauge invariance once a finite number of local counterterms are subtracted. In particular our (linearized) gauge invariance involves unconstrained fully symmetric parameters and is the same as the one considered in [4, 5, 39-41]. We are therefore naturally led to the problem of expressing our results in the geometric language of $[7,57]$. This is done in full generality in section 2 .

Another relevant issue is whether it is possible to construct a gauge invariant effective action without the subtraction of ad hoc non-invariant counterterms. The answer to this question of course requires the choice of a specific regularization scheme. We decided to work in dimensional regularization, which turns out to be particularly convenient for the lower spin cases. In fact, in section 3 we have explictly shown that for spin 1 and spin 2 gauge fields it is generally possible to introduce additional local terms allowed by covariance (involving the spin 0 current) such that the effective action is gauge invariant with no non-covariant subtractions needed thanks to the tadpole and seagull diagrams entering the Ward Identities. This is no surprise, as in these cases we already know fully off-shell covariant versions of QED and gravity coupled to ordinary scalar and spin $1 / 2$ matter. Nevertheless our explict computations work as a promising test for higher spin gauge fields. Related questions are whether this whole procedure depends on the choice of 
the on-shell conserved current that is minimally coupled in the first place and whether it can be restricted to a finite number of higher spin currents. In the cases of spin 1 and 2 we checked there is no need to introduce higher spin currents and and off-shell invariant couplings between the matter and gauge sectors can be obtained for any choice of on-shell conserved Noether currents. A particularly interesting choice for the currents and the couplings for the spin 2 case in $d=4$ is the one for which the dominating term in the UV expansion is the Weyl density $((3.54),(3.81))$, corresponding to the emerging conformal symmetry in the massless case. This term is also found in the corresponding IR expansion ((3.52), (3.77)). The case of higher spin gauge fields, in particular as far as the choice of different currents is concerned, will be treated in details in a subsequent work.

The major task of this paper was the completion of the construction of the quadratic part of the higher spin effective action started in $[1,42]$. We did it in section 4 presenting all two point correlators of symmetric currents of any spin up to 5 and in any dimension between 3 and 6 . We also spelled out UV and IR expansions, finding compact formulae for dominating terms in the two limits. In some cases these limits are already known [66]. In particular, we also included the mixed ones which were not considered in our previous work. The results of section 4 show that these terms turn out to have the usual structure found for the diagonal ones, i.e. the sum of a nonlocal transverse part and a local longitudinal one. We expect that their presence is crucial when one tries to test the invariance of the effective action beyond the lowest order in the gauge fields as well as when one tries to introduce tadpoles and seagulls in the Ward Identities. In $d=3$ odd-parity kinetic terms are present when spin $1 / 2$ matter is integrated out. We find that for the traceless currents considered in section 4 the UV limit coincides with a mixed-spin generalization of the conformal higher spin action found in $[7,8]$. Recently, supersymmetric generalizations have been discussed [14-16], pointing out dualities and extension to massive higher spin fields. It would be interesting to use the method of induced action to find back these results and possibly extend them to other cases.

Although made up of the same invariant building blocks, the kinetic terms appearing in this section do not coincide with the ones found in $[4,5,39]$ by coupling the gauge theory to matter and considering the analysis of the propagating degrees of freedom. The fundamental reason is that our effective actions are obtained after a subtraction procedure that is required to give invariant terms, but still allows for a wide class of possible choices. A proper discussion of the propagating degrees of freedom in our case would require the removal of nonlocalities by introducing compensating fields and the consideration of physical propagators after proper gauge fixing. However, the next logical step is the computation of three-point functions which would provide an insight in gauge invariance beyond the lowest order and therefore prepare the ground for the construction of fully covariant effective actions.

To end the section we would like to say a few words to explain the difference of our treatment of the effective action, as tackled in this paper, with other approaches. A particularly interesting one is that of [24] (although the main focus of [24] is in conformal higher spin theories). To this purpose the author utilizes a quantum mechanical approach of a particle coupled to external symmetric fields, that makes essential use of Weyl quantization 
and the relevant Moyal product. This method is equivalent to considering the effective action of a massless scalar field coupled to external sources. It allows one to define an infinite set of (traceless) conserved Noether currents, coupled to the external (symmetric) higher spin fields, as well as the relevant gauge symmetries. It allows also to compute the relevant quadratic effective action of the external fields and, at least in principle, the effective action to all orders. As said above, the interest of [24] is limited to conformal higher spin theories. Later on Bekaert et al. [25] have revisited and extended this method, but always considering a massless scalar.

As compared to [24, 25], our method is based on Feynman diagrams together with a considerable technical improvement in computing the latter. In particular, in the present draft, we have extended our previous results in [1] to mixed spin terms, which also turn out to be always made up of a nonlocal transverse part and a local non-transverse one. The main difference with the methods of [24, 25], is that we deal with massive scalar and fermion theories coupled to external sources. The introduction of a mass is important because it allows us to intercept not only the conformal higher spin kinetic terms which can be found in [66], but also the Fronsdal equations for symmetric fields which appear in $[4,5]$. It also allows to know the nonlocal part of the effective action, and to appreciate the fact that the latter is based on the same type of Fronsdal operators, as it is explained in section 2. Continuing with the differences with [24], the introduction of a mass term breaks Weyl invariance. Therefore, our conserved currents cannot in general be chosen to be traceless. In any case, consistently with the general Noether procedure, we have several choices for the conserved currents and the corresponding lowest order gauge symmetries. It is of course interesting to analyze the differences between different choices, which we plan to discuss in future work. As for the gauge symmetry, once a current is chosen its form is self-evident at the lowest order of approximation, but in order to compute the next orders, the procedure is more complicated. In our effective field theory approach we can also read off the needed seagull counterterms directly from the amplitudes by requiring the vanishing of non-transverse parts. But, of course, nothing prevents from merging the two methods. This is precisely what we would like to do in the future.

\section{Acknowledgments}

We would like to thank Nicolas Boulanger and Sergei M. Kuzenko for useful suggestions in revising the manuscript. This research has been supported by the Croatian Science Foundation under the project No. 8946 and by the University of Rijeka under the research support No. 13.12.1.4.05.

\section{A Spin 2 - expansions}

Here, we list several useful expansions of geometrical quantities in terms of spin 2 field $h_{\mu \nu}$.

Riemann tensor

$$
R_{\mu \nu \lambda \rho}=-\frac{1}{2} \partial_{\mu} \partial_{\lambda} h_{\nu \rho}+\frac{1}{2} \partial_{\mu} \partial_{\rho} h_{\nu \lambda}+\frac{1}{2} \partial_{\nu} \partial_{\lambda} h_{\mu \rho}-\frac{1}{2} \partial_{\nu} \partial_{\rho} h_{\mu \lambda}+O\left(h^{2}\right)
$$


Ricci tensor

$$
R_{\mu \nu}=-\frac{1}{2} \partial_{\mu} \partial_{\nu} h+\frac{1}{2} \partial_{\mu} \partial_{\lambda} h_{\nu}^{\lambda}+\frac{1}{2} \partial_{\nu} \partial_{\lambda} h_{\mu}^{\lambda}-\frac{1}{2} \square h_{\mu \nu}+O\left(h^{2}\right)
$$

Ricci scalar

$$
\begin{aligned}
R= & -\square h+\partial_{\mu} \partial_{\nu} h^{\mu \nu} \\
& +h^{\mu \nu} \partial_{\mu} \partial_{\nu} h-2 h^{\mu \nu} \partial_{\nu} \partial_{\lambda} h_{\mu}^{\lambda}-\frac{1}{4} \partial_{\nu} h \partial^{\nu} h-\partial_{\nu} h^{\mu \nu} \partial_{\lambda} h_{\mu}^{\lambda}+\partial_{\mu} h \partial_{\nu} h^{\mu \nu}+h^{\mu \nu} \square h_{\mu \nu} \\
& -\frac{1}{2} \partial_{\lambda} h^{\mu \nu} \partial_{\nu} h_{\mu}^{\lambda}+\frac{3}{4} \partial_{\lambda} h_{\mu \nu} \partial^{\lambda} h^{\mu \nu}+O\left(h^{3}\right)
\end{aligned}
$$

Einstein-Hilbert

$$
\begin{aligned}
& \sqrt{g} R=-\square h+\partial_{\mu} \partial_{\nu} h^{\mu \nu}+\frac{1}{2}\left(h^{\mu \nu} \square h_{\mu \nu}+2 h^{\mu \nu} \partial_{\mu} \partial_{\nu} h-h \square h-2 h^{\mu \lambda} \partial_{\mu} \partial_{\lambda} h_{\nu}^{\lambda}\right)+O\left(h^{3}\right) \\
& \sqrt{g} R \leftrightarrow k^{2} \pi_{\mu \mu} h^{\mu \mu}+h^{\mu \mu}\left(-\frac{1}{4} k^{2}\left(\pi_{\mu \nu}^{2}-\pi_{\mu \mu} \pi_{\nu \nu}\right)\right) h^{\nu \nu}+O\left(h^{3}\right)
\end{aligned}
$$

Riemann squared

$$
\begin{aligned}
R_{\kappa \lambda \rho \sigma} R^{\kappa \lambda \rho \sigma} & =h^{\mu \nu} \partial_{\mu} \partial_{\nu} \partial_{\lambda} \partial_{\rho} h^{\lambda \rho}-2 h^{\mu \nu} \partial_{\mu} \partial_{\lambda} \square h_{\nu}^{\lambda}+h^{\mu \nu} \square^{2} h_{\mu \nu}+O\left(h^{3}\right) \\
\sqrt{g} R_{\kappa \lambda \rho \sigma} R^{\kappa \lambda \rho \sigma} & \leftrightarrow h^{\mu \mu}\left(k^{4} \pi_{\mu \nu}^{2}\right) h^{\nu \nu}+O\left(h^{3}\right)
\end{aligned}
$$

Ricci tensor squared

$$
\begin{aligned}
R_{\mu \nu} R^{\mu \nu}= & \frac{1}{4} h^{\mu \nu} \square^{2} h_{\mu \nu}-\frac{1}{2} h^{\mu \nu} \partial_{\mu} \partial_{\nu} \square h-\frac{1}{2} h^{\mu \nu} \partial_{\mu} \partial_{\lambda} \square h_{\nu}^{\lambda} \\
& +\frac{1}{2} h^{\mu \nu} \partial_{\mu} \partial_{\nu} \partial_{\lambda} \partial_{\rho} h^{\lambda \rho}+\frac{1}{4} h \square^{2} h+O\left(h^{3}\right) \\
\sqrt{g} R_{\kappa \lambda} R^{\kappa \lambda} \leftrightarrow & h^{\mu \mu}\left(\frac{1}{4} k^{4}\left(\pi_{\mu \nu}^{2}+\pi_{\mu \mu} \pi_{\nu \nu}\right)\right) h^{\nu \nu}+O\left(h^{3}\right)
\end{aligned}
$$

Ricci scalar squared

$$
\begin{aligned}
R^{2} & =h^{\mu \nu} \partial_{\mu} \partial_{\nu} \partial_{\lambda} \partial_{\rho} h^{\lambda \rho}-2 h^{\mu \nu} \partial_{\mu} \partial_{\nu} \square h+h \square^{2} h+O\left(h^{3}\right) \\
\sqrt{g} R^{2} & \leftrightarrow h^{\mu \mu}\left(k^{4} \pi_{\mu \mu} \pi_{\nu \nu}\right) h^{\nu \nu}+O\left(h^{3}\right)
\end{aligned}
$$

Weyl density

$$
\begin{aligned}
W_{\mu \nu \rho \sigma}= & R_{\mu \nu \rho \sigma}-\frac{1}{d-2}\left(R_{\mu \rho} g_{\nu \sigma}-R_{\mu \sigma} g_{\nu \rho}-R_{\nu \rho} g_{\mu \sigma}+R_{\nu \sigma} g_{\mu \rho}\right)+ \\
& +\frac{R}{(d-1)(d-2)}\left(g_{\mu \rho} g_{\nu \sigma}-g_{\mu \sigma} g_{\nu \rho}\right) \\
W_{\mu \nu \rho \sigma} W^{\mu \nu \rho \sigma}= & R_{\mu \nu \rho \sigma} R^{\mu \nu \rho \sigma}-\frac{4}{d-2} R_{\mu \nu} R^{\mu \nu}+\frac{2}{d^{2}-3 d+2} R^{2} \\
\sqrt{g} W_{\kappa \lambda \rho \sigma} W^{\kappa \lambda \rho \sigma} \leftrightarrow & h^{\mu \mu}\left(\frac{d-3}{d-2} k^{4} \pi_{\mu \nu}^{2}-\frac{d-3}{(d-1)(d-2)} k^{4} \pi_{\mu \mu} \pi_{\nu \nu}\right) h^{\nu \nu}+O\left(h^{3}\right)
\end{aligned}
$$


Weyl density for $d=4$

$$
\begin{aligned}
\mathcal{W}^{2} & =R_{\mu \nu \rho \sigma} R^{\mu \nu \rho \sigma}-2 R_{\mu \nu} R^{\mu \nu}+\frac{1}{3} R^{2} \\
& =\frac{1}{2} h^{\mu \nu} \square^{2} h_{\mu \nu}-h^{\mu \nu} \partial_{\mu} \partial_{\lambda} \square h_{\nu}^{\lambda}+\frac{1}{3} h^{\mu \nu} \partial_{\mu} \partial_{\nu} \square h+\frac{1}{3} h^{\mu \nu} \partial_{\mu} \partial_{\nu} \partial_{\lambda} \partial_{\rho} h^{\lambda \rho}-\frac{1}{6} h \square^{2} h+O\left(h^{3}\right)
\end{aligned}
$$

Cotton tensor for $d=3$

$$
C_{\mu \nu}=\epsilon_{\mu}^{\tau \rho} \partial_{\tau}\left(R_{\rho \nu}-\frac{1}{d-1} g_{\nu \rho} R\right)=\frac{1}{2} \epsilon_{\mu}^{\rho \tau} \partial_{\tau}\left(\square h_{\nu \rho}-\partial_{\lambda} \partial_{\nu} h_{\rho}^{\lambda}\right)+O\left(h^{2}\right)
$$

\section{B Higher spin traceless actions}

In this appendix we will review the parity-odd actions that are expected in 3D for conformal higher spin in Minkowski background, showing that they coincide with the UV limits of the amplitudes considered in sections 3.1.1, 3.3.2, 4.1-4.3. ${ }^{6}$ The action considered in [8] can be easily generalized to the case when the quadratic terms involve fields of different spins, namely

$$
\begin{aligned}
I_{s_{1}, s_{2}}= & \frac{1}{2} \int d^{3} x \sum_{r=0}^{s_{1}-1}\left(\begin{array}{c}
2 s_{1} \\
2 r+1
\end{array}\right)^{\left(s_{1}\right)^{\alpha_{1} \ldots \alpha_{2 s_{1}}}}(\square)^{r} \partial_{\alpha_{1}}^{\beta_{1}} \ldots \partial_{\alpha_{2 s_{1}-2 r-1}} \beta_{2 s_{1}-2 r-1} \\
& \partial^{\beta_{2 s_{1}+1} \beta_{2 s_{1}+2}} \ldots \partial^{\beta_{2 s_{2}-1} \beta_{2 s_{2}}}{\stackrel{\left(s_{2}\right)}{h}}_{\beta_{1} \ldots \beta_{2 s_{1}-2 r-1} \alpha_{2 s_{1}-2 r} \ldots \alpha_{2 s_{1}} \beta_{2 s_{1}+1} \ldots \beta_{2 s_{2}}},
\end{aligned}
$$

where we assume $s_{1} \leq s_{2}$ and $\partial_{\alpha}^{\beta}=\left(\gamma^{\mu}\right)_{\alpha}^{\beta} \partial_{\mu}$. We define $\left(\gamma_{\mu}\right)_{\alpha \beta}=\varepsilon_{\beta \gamma}\left(\gamma_{\mu}\right)_{\alpha}^{\gamma}$ and $\left(\gamma_{\mu}\right)^{\alpha \beta}=\varepsilon^{\alpha \gamma}\left(\gamma_{\mu}\right)_{\gamma}{ }^{\beta}$, in agreement with the conventions of Wess and Bagger. Going from the spinor notation $h^{\alpha_{1} \ldots \alpha_{2 s}}=h^{\mu_{1} \ldots \mu_{s}}\left(\gamma_{\mu_{1}}\right)^{\alpha_{1} \alpha_{2}} \ldots\left(\gamma_{\mu_{s}}\right)^{\alpha_{2 s-1} \alpha_{2 s}}$ to the standard tensor one, we get

$$
\begin{aligned}
I_{s_{1}, s_{2}}= & \frac{1}{2} \int d^{3} x \sum_{r=0}^{s_{1}-1}\left(\begin{array}{c}
2 s_{1} \\
2 r+1
\end{array}\right) \stackrel{\left(s_{1}\right)^{\mu_{1} \ldots \mu_{s_{1}}}}{h}\left(\gamma_{\mu_{1}}\right)^{\alpha_{1} \alpha_{2}} \ldots\left(\gamma_{\mu_{s_{1}}}\right)^{\alpha_{2 s_{1}-1} \alpha_{2 s_{1}}}(\square)^{r} \\
& \partial_{\alpha_{1}}^{\beta_{1}} \ldots \partial_{\alpha_{2 s_{1}-2 r-1}}^{\beta_{2 s_{1}-2 r-1}} \delta_{\alpha_{2 s_{1}-2 r}}^{\beta_{2 s_{1}-2 r}} \ldots \delta_{\alpha_{2 s_{1}}}^{\beta_{2 s_{1}}} \partial^{\beta_{2 s_{1}+1} \beta_{2 s_{1}+2}} \ldots \partial^{\beta_{2 s_{2}-1} \beta_{2 s_{2}}} \\
& \left(\gamma_{\nu_{1}}\right)_{\beta_{1} \beta_{2}} \ldots\left(\gamma_{\nu_{s_{2}}}\right)_{\beta_{2 s_{2}-1} \beta_{2 s_{2}}} \stackrel{\left.s_{2}\right)^{\nu_{1} \ldots \nu_{s_{2}}}}{h} \\
= & \frac{1}{2} \int d^{3} x \sum_{r=0}^{s_{1}-1}\left(\begin{array}{c}
2 s_{1} \\
2 r+1
\end{array}\right) \stackrel{\left(s_{1}\right)^{\mu_{1} \ldots \mu_{s_{1}}}}{h}(\square)^{r}\left[\left(\gamma_{\mu_{1}}\right)^{\alpha_{1} \alpha_{2}}{\partial_{\alpha_{1}}}^{\beta_{1}}\left(\gamma_{\nu_{1}}\right)_{\beta_{1} \beta_{2}} \partial_{\alpha_{2}}^{\beta_{2}}\right] \ldots \\
& \ldots\left[\left(\gamma_{\mu_{s_{1}-r-1}}\right)^{\alpha_{2 s_{1}-2 r-3} \alpha_{2 s_{1}-2 r-2}} \partial_{\alpha_{2 s_{1}-2 r-3}}^{\beta_{2 s_{1}-2 r-3}}\right. \\
& \left.\left(\gamma_{\nu_{s_{1}-r-1}}\right)_{\beta_{2 s_{1}-2 r-3} \beta_{2 s_{1}-2 r-2}} \partial_{\alpha_{2 s_{1}-2 r-2}}^{\beta_{2 s_{1}-2 r-2}}\right] \\
& {\left[\left(\gamma_{\mu_{s_{1}-r}}\right)^{\alpha_{2 s_{1}-2 r-1} \alpha_{2 s_{1}-2 r}} \partial_{\alpha_{2 s_{1}-2 r-1}}^{\beta_{2 s_{1}-2 r-1}}\left(\gamma_{\nu_{s_{1}-r}}\right)_{\beta_{2 s_{1}-2 r-1} \beta_{2 s_{1}-2 r}} \delta_{\alpha_{2 s_{1}-2 r}}^{\beta_{2 s_{1}-2 r}}\right] }
\end{aligned}
$$

\footnotetext{
${ }^{6}$ It is understood the UV limit should be carried out as described in [43], introducing an ad hoc flavor index.
} 


$$
\begin{aligned}
& {\left[\left(\gamma_{\mu_{s_{1}-r+1}}\right)^{\alpha_{2 s_{1}-2 r+1} \alpha_{2 s_{1}-2 r+2}}\left(\gamma_{\nu_{s-r+1}}\right)_{\beta_{2 s-2 r+1} \beta_{2 s-2 r+2}} \delta_{\alpha_{2 s-2 r+1}}^{\beta_{2 s_{1}-2 r+1}} \delta_{\alpha_{2 s_{1}-2 r+2}}^{\beta_{2 s_{1}-2 r+2}}\right] \ldots} \\
& \ldots\left[\left(\gamma_{\mu_{1}}\right)^{\alpha_{2 s_{1}-1} \alpha_{2 s_{1}}}\left(\gamma_{\nu_{s_{1}}}\right)_{\beta_{2 s_{1}-1} \beta_{2 s_{1}}} \delta_{\alpha_{2 s_{1}-1} \beta_{2 s_{1}} \delta_{\alpha_{2}}}^{\beta_{2 s_{1}}}\right] \\
& {\left[\partial^{\beta_{2 s_{1}+1} \beta_{2 s_{1}+2}}\left(\gamma_{\nu_{s_{1}+1}}\right)_{\beta_{2 s_{1}+1} \beta_{2 s_{1}+2}}\right] \ldots\left[\partial^{\beta_{2 s_{2}-1} \beta_{2 s_{2}}}\left(\gamma_{\nu_{s_{2}}}\right)_{\beta_{2 s_{2}-1} \beta_{2 s_{2}}}\right] \stackrel{\left(s_{2}\right)^{\nu_{1} \ldots \nu_{s}}}{h}}
\end{aligned}
$$

where, keeping in mind that in $D=3\left(\gamma_{\mu}\right)_{\alpha \beta}=\left(\gamma_{\mu}\right)_{\beta \alpha}$, we can easily recognize the traces

$$
\begin{aligned}
\operatorname{Tr}\left[\gamma_{\mu_{i}}(\gamma \cdot \partial) \gamma_{\nu_{i}}(\gamma \cdot \partial)\right]= & {\left[\left(\gamma_{\mu_{i}}\right)^{\alpha_{2 i-1} \alpha_{2 i}} \partial_{\alpha_{2 i-1}}^{\beta_{2 i-1}}\left(\gamma_{\nu_{i}}\right)_{\beta_{2 i-1} \beta_{2 i}} \partial_{\alpha_{2 i}}{ }^{\beta_{2 i}}\right], } \\
-\operatorname{Tr}\left[\gamma_{\mu_{s_{1}-r}}(\gamma \cdot \partial) \gamma_{\nu_{s_{1}-r}}\right]= & {\left[\left(\gamma_{\mu_{s_{1}-r}}\right)^{\alpha_{2 s_{1}-2 r-1} \alpha_{2 s_{1}-2 r}} \partial_{\alpha_{2 s_{1}-2 r-1}} \beta_{2 s_{1}-2 r-1}\right.} \\
& \left.\left(\gamma_{\nu_{s_{1}-r}}\right)_{\beta_{2 s_{1}-2 r-1} \beta_{2 s_{1}-2 r}} \delta_{\alpha_{2 s_{1}-2 r}}^{\beta_{2 s_{1}-2 r}}\right], \\
-\operatorname{Tr}\left[\gamma_{\mu_{j}} \gamma_{\nu_{j}}\right]= & {\left[\left(\gamma_{\mu_{j}}\right)^{\alpha_{2 j-1} \alpha_{2 j}}\left(\gamma_{\nu_{j}}\right)_{\beta_{2 j-1} \beta_{2 j}} \delta_{\alpha_{2 j-1}}^{\beta_{2 j-1}} \delta_{\alpha_{2 j}}^{\beta_{2 j}}\right], } \\
-\operatorname{Tr}\left[(\gamma \cdot \partial) \gamma_{\nu_{k}}\right]= & {\left[\partial^{\beta_{2 k-1} \beta_{2 k}}\left(\gamma_{\nu_{k}}\right)_{\beta_{2 k-1} \beta_{2 k}}\right] . }
\end{aligned}
$$

In order to evaluate these traces, we need the following rules

$$
\begin{aligned}
\operatorname{Tr}\left[\gamma_{\mu} \gamma_{\nu}\right] & =2 \eta_{\mu \nu}, \\
\operatorname{Tr}\left[\gamma_{\mu} \gamma_{\nu} \gamma_{\rho}\right] & =-2 \varepsilon_{\mu \nu \rho}, \\
\operatorname{Tr}\left[\gamma_{\mu} \gamma_{\nu} \gamma_{\rho} \gamma_{\sigma}\right] & =2\left(\eta_{\mu \nu} \eta_{\rho \sigma}-\eta_{\mu \rho} \eta_{\nu \sigma}+\eta_{\mu \sigma} \eta_{\nu \rho}\right),
\end{aligned}
$$

which imply

$$
\begin{aligned}
\operatorname{Tr}\left[(\gamma \cdot \partial) \gamma_{\nu_{k}}\right] & =2 \partial_{\nu_{k}} \\
\operatorname{Tr}\left[\gamma_{\mu_{s-r}}(\gamma \cdot \partial) \gamma_{\nu_{s-r}}\right] & =2 \varepsilon_{\mu_{s-r} \nu_{s-r} \rho} \partial^{\rho} \\
\operatorname{Tr}\left[\gamma_{\mu_{i}}(\gamma \cdot \partial) \gamma_{\nu_{i}}(\gamma \cdot \partial)\right] & =2\left(2 \partial_{\mu_{i}} \partial_{\nu_{i}}-\eta_{\mu_{i} \nu_{i}} \square\right)
\end{aligned}
$$

We can therefore rewrite the action $I_{s_{1}, s_{2}}$ as

$$
\begin{aligned}
& I_{s_{1}, s_{2}}=\frac{1}{2}(-2)^{s_{2}} \int d^{3} x \sum_{r=0}^{s_{1}-1}\left(\begin{array}{c}
2 s_{1} \\
2 r+1
\end{array}\right) \stackrel{\left(s_{1}\right)^{\mu_{1} \ldots \mu_{s_{1}}}}{h}(\square)^{r}\left(\eta_{\mu_{1} \nu_{1}} \square-2 \partial_{\mu_{1}} \partial_{\nu_{1}}\right) \ldots \\
& \ldots\left(\eta_{\mu_{s_{1}-r-1} \nu_{s_{1}-r-1}} \square-2 \partial_{\mu_{s_{1}-r-1}} \partial_{\nu_{s_{1}-r-1}}\right) \varepsilon_{\mu_{s_{1}-r} \nu_{s_{1}-r} \rho} \partial^{\rho}
\end{aligned}
$$

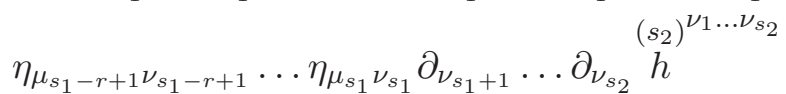

$$
\begin{aligned}
& =-(-2)^{s_{2}-1} \int d^{3} x \sum_{r=0}^{s_{1}-1}\left(\begin{array}{c}
2 s_{1} \\
2 r+1
\end{array}\right) \stackrel{\left(s_{1}\right)^{\mu_{1} \ldots \mu_{s_{1}}}}{h}(\square)^{r} \varepsilon_{\mu_{1} \nu_{1} \rho} \partial^{\rho} \eta_{\mu_{2} \nu_{2}} \ldots \eta_{\mu_{r+1} \nu_{r+1}} \\
& \left(\eta_{\mu_{r+2} \nu_{r+2}} \square-2 \partial_{\mu_{r+2}} \partial_{\nu_{r+2}}\right) \ldots\left(\eta_{\mu_{s_{1}} \nu_{s_{1}}} \square-2 \partial_{\mu_{s_{1}}} \partial_{\nu_{s_{1}}}\right) \\
& \partial_{\nu_{s_{1}+1}} \ldots \partial_{\nu_{s_{2}}} \stackrel{\left(s_{2}\right)^{\nu_{1} \ldots \nu_{s_{2}}}}{h} \text {. }
\end{aligned}
$$


By elementary manipulations, one can write the action (B.2) as a triple summation

$$
\begin{aligned}
& I_{s_{1}, s_{2}}=-(-2)^{s_{2}-1} \int d^{3} x h^{\left(s_{1}\right)^{\mu_{1} \ldots \mu_{s_{1}}}} \varepsilon_{\mu_{1} \nu_{1} \rho} \partial^{\rho} \sum_{r=0}^{s_{1}-1} \sum_{j=0}^{s_{1}-r-1} \sum_{k=0}^{s_{1}-j-1} \\
& (-2)^{j}\left(\begin{array}{c}
2 s_{1} \\
2 r+1
\end{array}\right)\left(\begin{array}{c}
s_{1}-r-1 \\
j
\end{array}\right)\left(\begin{array}{c}
s_{1}-j-1 \\
k
\end{array}\right)\left(\eta_{\mu_{2} \nu_{2}} \square-\partial_{\mu_{2}} \partial_{\nu_{2}}\right) \ldots \\
& \left(\eta_{\mu_{k+1} \nu_{k+1}} \square-\partial_{\mu_{k+1}} \partial_{\nu_{k+1}}\right)\left(\partial_{\mu_{k+2}} \partial_{\nu_{k+2}}\right) \ldots\left(\partial_{\mu_{s_{1}}} \partial_{\nu_{s_{1}}}\right) \partial_{\nu_{s_{1}+1}} \ldots \partial_{\nu_{s_{2}}} \stackrel{\left(s_{2}\right)^{\nu_{1} \ldots \nu_{s_{2}}}}{h} .
\end{aligned}
$$

We can now switch the order of summation

$$
\begin{aligned}
& I_{s_{1}, s_{2}}=-(-2)^{s_{2}-1} \int d^{3} x h^{\left(s_{1}\right)^{\mu_{1} \ldots \mu_{s_{1}}}} \varepsilon_{\mu_{1} \nu_{1} \rho} \partial^{\rho} \sum_{k=0}^{s_{1}-1}(-)^{s_{1}-k-1} \sum_{j=0}^{s_{1}-k-1} \sum_{r=0}^{s_{1}-j-1} \\
& (-2)^{j}\left(\begin{array}{c}
2 s_{1} \\
2 r+1
\end{array}\right)\left(\begin{array}{c}
s_{1}-r-1 \\
j
\end{array}\right)\left(\begin{array}{c}
s_{1}-j-1 \\
k
\end{array}\right)\left(\eta_{\mu_{2} \nu_{2}} \square-\partial_{\mu_{2}} \partial_{\nu_{2}}\right) \ldots \\
& \left(\eta_{\mu_{k+1} \nu_{k+1}} \square-\partial_{\mu_{k+1}} \partial_{\nu_{k+1}}\right)\left(-\partial_{\mu_{k+2}} \partial_{\nu_{k+2}}\right) \ldots\left(-\partial_{\mu_{s_{1}}} \partial_{\nu_{s_{1}}}\right) \partial_{\nu_{s_{1}+1}} \ldots \partial_{\nu_{s_{2}}} \stackrel{\left(s_{2}\right)^{\nu_{1} \ldots \nu_{s_{2}}}}{h},
\end{aligned}
$$

and perform two summations using the tables or Mathematica

$$
\begin{aligned}
& \sum_{j=0}^{s_{1}-k-1} \sum_{r=0}^{s_{1}-j-1}(-2)^{j}\left(\begin{array}{c}
2 s_{1} \\
2 r+1
\end{array}\right)\left(\begin{array}{c}
s_{1}-r-1 \\
j
\end{array}\right)\left(\begin{array}{c}
s_{1}-j-1 \\
k
\end{array}\right) \\
& =2^{-1+2 s_{1}}\left(\begin{array}{c}
s_{1}-1 \\
k
\end{array}\right){ }_{2} F_{1}\left(\frac{1}{2}-s_{1}, 1+k-s_{1}, 1-2 s_{1} ; 2\right)
\end{aligned}
$$

thus obtaining

$$
\begin{aligned}
I_{s_{1}, s_{2}}= & -(-2)^{s_{2}-1} 2^{-1+2 s_{1}} \int d^{3} x \stackrel{\left(s_{1}\right)^{\mu_{1} \ldots \mu_{s_{1}}}}{h} \varepsilon_{\mu_{1} \nu_{1} \rho} \partial^{\rho} \sum_{k=0}^{s_{1}-1}(-)^{s_{1}-k-1}\left(\begin{array}{c}
s_{1}-1 \\
k
\end{array}\right) \\
& { }_{2} F_{1}\left(\frac{1}{2}-s_{1}, 1+k-s_{1}, 1-2 s_{1} ; 2\right)\left(\eta_{\mu_{2} \nu_{2}} \square-\partial_{\mu_{2}} \partial_{\nu_{2}}\right) \ldots \\
& \left(\eta_{\mu_{k+1} \nu_{k+1}} \square-\partial_{\mu_{k+1}} \partial_{\nu_{k+1}}\right)\left(-\partial_{\mu_{k+2}} \partial_{\nu_{k+2}}\right) \ldots\left(-\partial_{\mu_{s_{1}}} \partial_{\nu_{s_{1}}}\right) \partial_{\nu_{s_{1}+1}} \ldots \partial_{\nu_{s_{2}}} \stackrel{\left(s_{2}\right)^{\nu_{1} \ldots \nu_{s_{2}}}}{ }
\end{aligned}
$$

or equivalently

$$
\begin{aligned}
I_{s_{1}, s_{2}}= & -(-2)^{s_{2}-1} 2^{-1+2 s_{1}} \int d^{3} x \stackrel{\left(s_{1}\right)^{\mu_{1} \ldots \mu_{s_{1}}}}{h} \varepsilon_{\mu_{1} \nu_{1} \rho} \partial^{\rho} \sum_{k=0}^{s_{1}-1}(-)^{k}\left(\begin{array}{c}
s_{1}-1 \\
s_{1}-1-k
\end{array}\right) \\
& { }_{2} F_{1}\left(-k, \frac{1}{2}-s_{1}, 1-2 s_{1} ; 2\right)\left(-\partial_{\mu_{2}} \partial_{\nu_{2}}\right) \ldots\left(-\partial_{\mu_{k+1}} \partial_{\nu_{k+1}}\right) \\
& \left(\eta_{\mu_{k+2} \nu_{k+2}} \square-\partial_{\mu_{k+2}} \partial_{\nu_{k+2}}\right) \ldots\left(\eta_{\mu_{s_{1}} \nu_{s_{1}}} \square-\partial_{\mu_{s_{1}}} \partial_{\nu_{s_{1}}}\right) \partial_{\nu_{s_{1}+1}} \ldots \partial_{\nu_{s_{2}}} \stackrel{\left(s_{2}\right)^{\nu_{1} \ldots \nu_{s_{2}}}}{.}
\end{aligned}
$$

Using the recursion relation ${ }_{2} F_{1}\left(-k, \frac{1}{2}-s, 1-2 s ; 2\right)=\frac{2-2 s+k}{k+1}{ }_{2} F_{1}\left(-k-2, \frac{1}{2}-s, 1-2 s ; 2\right)$ and the starting values ${ }_{2} F_{1}\left(0, \frac{1}{2}-s, 1-2 s ; 2\right)=1$ and ${ }_{2} F_{1}\left(-1, \frac{1}{2}-s, 1-2 s ; 2\right)=0$, 
one finds

$$
\begin{aligned}
{ }_{2} F_{1}\left(-2 j-1, \frac{1}{2}-s, 1-2 s ; 2\right) & =0 \\
{ }_{2} F_{1}\left(-2 j, \frac{1}{2}-s, 1-2 s ; 2\right) & =\frac{\Gamma(2 j+1) \Gamma(1-2 s)}{\Gamma(2 j-2 s+1)} P_{k}^{\left(-2 s, s-2 j-\frac{1}{2}\right)} \\
& =\frac{\Gamma(2 j+1) \Gamma(1-2 s)}{\Gamma(2 j-2 s+1)}\left(\begin{array}{c}
j-s-\frac{1}{2} \\
j
\end{array}\right) \\
& =\frac{(-1)^{j}}{2^{2 j}} \frac{\Gamma(2 j) \Gamma(s-j)}{\Gamma(s) \Gamma(j)} .
\end{aligned}
$$

The summation is therefore only over even $k=2 j$ and, using the fact that $h$ 's are traceless and the convenient notation of projectors $\pi_{\mu \nu}=\eta_{\mu \nu}-\frac{\partial_{\mu} \partial_{\nu}}{\square}$. we can write down

$$
\begin{aligned}
I_{s_{1}, s_{2}}= & -(-2)^{s_{2}-1} 2^{-1+2 s_{1}} \int d^{3} x \stackrel{\left(s_{1}\right)^{\mu_{1} \ldots \mu_{s_{1}}}}{h} \varepsilon_{\mu_{1} \nu_{1} \rho} \partial^{\rho} \square^{s_{1}-1} \sum_{j=0}^{\left[\frac{s_{1}-1}{2}\right]}(-)^{j} \frac{1}{2^{2 j}}\left(\begin{array}{c}
s_{1}-1-j \\
j
\end{array}\right) \\
& \pi_{\mu_{2} \mu_{3}} \ldots \pi_{\mu_{2 j} \mu_{2 j+1}} \pi_{\nu_{2} \nu_{3}} \ldots \pi_{\nu_{2 j} \nu_{2 j+1}} \pi_{\mu_{2 j+2} \nu_{2 j+2}} \ldots \pi_{\mu_{s_{1}} \nu_{s_{1}}} \partial_{\nu_{s_{1}+1}} \ldots \partial_{\nu_{s_{2}}} \stackrel{\left(s_{2}\right)^{\nu_{1} \ldots \nu_{s_{2}}}}{h}
\end{aligned}
$$

One can go to momentum representation by the substitutions $\stackrel{(s)}{h}(x)=\int \frac{d^{3} k}{(2 \pi)^{3}} e^{-i k x} \stackrel{(s)}{h}(k)$,

$$
\begin{aligned}
I_{s_{1}, s_{2}}= & -(-2)^{s_{2}-1} 2^{-1+2 s_{1}}(-i)^{s_{2}-s_{1}+1} \\
& \int d^{3} k \stackrel{\left(s_{1}\right)^{\mu_{1} \ldots \mu_{s_{1}}}}{h} \varepsilon_{\mu_{1} \nu_{1} \rho} k^{\rho}\left(-k^{2}\right)^{s_{1}-1} \sum_{j=0}^{\left[\frac{s_{1}-1}{2}\right]}(-)^{j} \frac{1}{2^{2 j}}\left(\begin{array}{c}
s_{1}-1-j \\
j
\end{array}\right) \\
& \pi_{\mu_{2} \mu_{3}} \ldots \pi_{\mu_{2 j} \mu_{2 j+1}} \pi_{\nu_{2} \nu_{3}} \ldots \pi_{\nu_{2 j} \nu_{2 j+1}} \pi_{\mu_{2 j+2} \nu_{2 j+2}} \ldots \pi_{\mu_{s_{1}} \nu_{s_{1}}} k_{\nu_{s_{1}+1}} \ldots k_{\nu_{s_{2}}} \stackrel{\left(s_{2}\right)^{\nu_{1} \ldots \nu_{s_{2}}}}{h}
\end{aligned}
$$

which corresponds to the amplitude (4.14) up to an overall constant. This follows from the fact that $h$ is traceless and $s_{2}-s_{1}$ is even so we can substitute $k_{\nu_{s_{1}+1}} \ldots k_{\nu_{s_{2}}}$ with $\pi$ 's.

Open Access. This article is distributed under the terms of the Creative Commons Attribution License (CC-BY 4.0), which permits any use, distribution and reproduction in any medium, provided the original author(s) and source are credited.

\section{References}

[1] L. Bonora, M. Cvitan, P. Dominis Prester, S. Giaccari, B. Lima de Souza and T. Štemberga, One-loop effective actions and higher spins, JHEP 12 (2016) 084 [arXiv:1609.02088] [INSPIRE].

[2] C. Fronsdal, Massless Fields with Integer Spin, Phys. Rev. D 18 (1978) 3624 [InSPIRE].

[3] J. Fang and C. Fronsdal, Massless Fields with Half Integral Spin, Phys. Rev. D 18 (1978) 3630 [INSPIRE]. 
[4] D. Francia and A. Sagnotti, On the geometry of higher spin gauge fields, Class. Quant. Grav. 20 (2003) S473 [hep-th/0212185] [INSPIRE].

[5] D. Francia and A. Sagnotti, Free geometric equations for higher spins, Phys. Lett. B 543 (2002) 303 [hep-th/0207002] [INSPIRE].

[6] D.M. Capper, On quantum corrections to the graviton propagator, Nuovo Cim. A 25 (1975) 29 [inSPIRE].

[7] T. Damour and S. Deser, 'Geometry' of Spin 3 Gauge Theories, Ann. Inst. H. Poincare Phys. Theor. 47 (1987) 277.

[8] C.N. Pope and P.K. Townsend, Conformal Higher Spin in $(2+1)$-dimensions, Phys. Lett. B 225 (1989) 245 [inSPIRE].

[9] M. Henneaux, S. Hörtner and A. Leonard, Higher Spin Conformal Geometry in Three Dimensions and Prepotentials for Higher Spin Gauge Fields, JHEP 01 (2016) 073 [arXiv: 1511.07389] [INSPIRE].

[10] H. Linander and B.E.W. Nilsson, The non-linear coupled spin 2-spin 3 Cotton equation in three dimensions, JHEP 07 (2016) 024 [arXiv: 1602.01682] [INSPIRE].

[11] T. Basile, R. Bonezzi and N. Boulanger, The Schouten tensor as a connection in the unfolding of 3D conformal higher-spin fields, JHEP 04 (2017) 054 [arXiv:1701.08645] [INSPIRE].

[12] E.A. Bergshoeff, O. Hohm and P.K. Townsend, On Higher Derivatives in 3D Gravity and Higher Spin Gauge Theories, Annals Phys. 325 (2010) 1118 [arXiv:0911.3061] [InSPIRE].

[13] B.E.W. Nilsson, Towards an exact frame formulation of conformal higher spins in three dimensions, JHEP 09 (2015) 078 [arXiv:1312.5883] [INSPIRE].

[14] S.M. Kuzenko and D.X. Ogburn, Off-shell higher spin $N=2$ supermultiplets in three dimensions, Phys. Rev. D 94 (2016) 106010 [arXiv:1603.04668] [INSPIRE].

[15] S.M. Kuzenko, Higher spin super-Cotton tensors and generalisations of the linear-chiral duality in three dimensions, Phys. Lett. B 763 (2016) 308 [arXiv:1606.08624] [INSPIRE].

[16] S.M. Kuzenko and M. Tsulaia, Off-shell massive $N=1$ supermultiplets in three dimensions, Nucl. Phys. B 914 (2017) 160 [arXiv:1609.06910] [INSPIRE].

[17] A.D. Sakharov, Vacuum quantum fluctuations in curved space and the theory of gravitation, Sov. Phys. Dokl. 12 (1968) 1040 [INSPIRE].

[18] J.M. Maldacena and G.L. Pimentel, On graviton non-Gaussianities during inflation, JHEP 09 (2011) 045 [arXiv:1104.2846] [INSPIRE].

[19] X.O. Camanho, J.D. Edelstein, J. Maldacena and A. Zhiboedov, Causality Constraints on Corrections to the Graviton Three-Point Coupling, JHEP 02 (2016) 020 [arXiv:1407.5597] [INSPIRE].

[20] K.S. Babu, A.K. Das and P. Panigrahi, Derivative Expansion and the Induced Chern-Simons Term at Finite Temperature in (2+1)-dimensions, Phys. Rev. D 36 (1987) 3725 [INSPIRE].

[21] G.V. Dunne, Aspects of Chern-Simons theory, hep-th/9902115 [INSPIRE].

[22] J. Maldacena and A. Zhiboedov, Constraining Conformal Field Theories with A Higher Spin Symmetry, J. Phys. A 46 (2013) 214011 [arXiv:1112.1016] [InSPIRE]. 
[23] J. Maldacena and A. Zhiboedov, Constraining conformal field theories with a slightly broken higher spin symmetry, Class. Quant. Grav. 30 (2013) 104003 [arXiv:1204.3882] [InSPIRE].

[24] A.Y. Segal, Conformal higher spin theory, Nucl. Phys. B 664 (2003) 59 [hep-th/0207212] [INSPIRE].

[25] X. Bekaert, E. Joung and J. Mourad, Effective action in a higher-spin background, JHEP 02 (2011) 048 [arXiv: 1012.2103] [INSPIRE].

[26] F.S. Gama, J.R. Nascimento and A. Yu. Petrov, Derivative expansion and the induced Chern-Simons term in $N=1, D=3$ superspace, Phys. Rev. D 93 (2016) 045015 [arXiv: 1511.05471] [INSPIRE].

[27] C. Closset, T.T. Dumitrescu, G. Festuccia, Z. Komargodski and N. Seiberg, Comments on Chern-Simons Contact Terms in Three Dimensions, JHEP 09 (2012) 091 [arXiv: 1206.5218] [INSPIRE].

[28] S. Giombi, S. Prakash and X. Yin, A note on CFT correlators in three dimensions, JHEP 07 (2013) 105 [arXiv: 1104.4317] [INSPIRE].

[29] S. Giombi, S. Minwalla, S. Prakash, S.P. Trivedi, S.R. Wadia and X. Yin, Chern-Simons Theory with Vector Fermion Matter, Eur. Phys. J. C 72 (2012) 2112 [arXiv:1110.4386] [INSPIRE].

[30] A. Campoleoni, Higher Spins in D =2 +1, Subnucl. Ser. 49 (2013) 385 [arXiv:1110.5841] [INSPIRE].

[31] R. Argurio, G. Barnich, G. Bonelli and M. Grigoriev eds., Higher-Spin Gauge Theories, Proceedings of the First Solvay Workshop, Brussels Belgium (2004), International Solvay Institutes, Brussels Belgium (2006).

[32] D. Sorokin, Introduction to the classical theory of higher spins, AIP Conf. Proc. 767 (2005) 172 [hep-th/0405069] [INSPIRE].

[33] D. Francia and A. Sagnotti, Higher-spin geometry and string theory, J. Phys. Conf. Ser. 33 (2006) 57 [hep-th/0601199] [inSPIRE].

[34] A. Fotopoulos and M. Tsulaia, Gauge Invariant Lagrangians for Free and Interacting Higher Spin Fields. A Review of the BRST formulation, Int. J. Mod. Phys. A 24 (2009) 1 [arXiv:0805.1346] [INSPIRE].

[35] C. Iazeolla, On the Algebraic Structure of Higher-Spin Field Equations and New Exact Solutions, arXiv:0807.0406 [INSPIRE].

[36] A. Campoleoni, Metric-like Lagrangian Formulations for Higher-Spin Fields of Mixed Symmetry, Riv. Nuovo Cim. 33 (2010) 123 [arXiv:0910.3155] [INSPIRE].

[37] A. Sagnotti, Higher Spins and Current Exchanges, PoS (CORFU2011) 106 [arXiv: 1002.3388] [INSPIRE].

[38] D. Francia, Low-spin models for higher-spin Lagrangians, Prog. Theor. Phys. Suppl. 188 (2011) 94 [arXiv:1103.0683] [InSPIRE].

[39] D. Francia, J. Mourad and A. Sagnotti, Current Exchanges and Unconstrained Higher Spins, Nucl. Phys. B 773 (2007) 203 [hep-th/0701163] [INSPIRE].

[40] D. Francia, On the Relation between Local and Geometric Lagrangians for Higher spins, J. Phys. Conf. Ser. 222 (2010) 012002 [arXiv:1001.3854] [INSPIRE]. 
[41] D. Francia, Generalised connections and higher-spin equations, Class. Quant. Grav. 29 (2012) 245003 [arXiv:1209.4885] [INSPIRE].

[42] L. Bonora, M. Cvitan, P. Dominis Prester, B. Lima de Souza and I. Smolić, Massive fermion model in $3 d$ and higher spin currents, JHEP 05 (2016) 072 [arXiv: 1602.07178] [INSPIRE].

[43] L. Bonora et al., Massive Dirac Field in 3D and Induced Equations for Higher Spin Fields, in Proceedings of Group 31, Rio de Janeiro Brazil (2016).

[44] S. Giombi and X. Yin, Higher Spins in AdS and Twistorial Holography, JHEP 04 (2011) 086 [arXiv: 1004.3736] [INSPIRE].

[45] V.E. Didenko and E.D. Skvortsov, Exact higher-spin symmetry in CFT: all correlators in unbroken Vasiliev theory, JHEP 04 (2013) 158 [arXiv:1210.7963] [INSPIRE].

[46] C. Sleight and M. Taronna, Higher Spin Interactions from Conformal Field Theory: The Complete Cubic Couplings, Phys. Rev. Lett. 116 (2016) 181602 [arXiv:1603.00022] [INSPIRE].

[47] R. Bonezzi, N. Boulanger, D. De Filippi and P. Sundell, Noncommutative Wilson lines in higher-spin theory and correlation functions of conserved currents for free conformal fields, J. Phys. A 50 (2017) 475401 [arXiv:1705.03928] [InSPIRE].

[48] X. Bekaert, N. Boulanger and P. Sundell, How higher-spin gravity surpasses the spin two barrier: no-go theorems versus yes-go examples, Rev. Mod. Phys. 84 (2012) 987 [arXiv: 1007.0435] [INSPIRE].

[49] S. Weinberg, The Quantum Theory of Fields. Vol. 1: Foundations, Cambridge University Press, Cambridge U.K. (1995).

[50] M.A. Vasiliev, Consistent equation for interacting gauge fields of all spins in (3+1)-dimensions, Phys. Lett. B 243 (1990) 378 [InSPIRE].

[51] M.A. Vasiliev, Properties of equations of motion of interacting gauge fields of all spins in $(3+1)$-dimensions, Class. Quant. Grav. 8 (1991) 1387 [INSPIRE].

[52] M.A. Vasiliev, Algebraic aspects of the higher spin problem, Phys. Lett. B 257 (1991) 111 [INSPIRE].

[53] M.A. Vasiliev, More on equations of motion for interacting massless fields of all spins in (3+1)-dimensions, Phys. Lett. B 285 (1992) 225 [INSPIRE].

[54] T. Curtright, Massless Field Supermultiplets With Arbitrary Spin, Phys. Lett. 85B (1979) 219 [INSPIRE].

[55] L.P.S. Singh and C.R. Hagen, Lagrangian formulation for arbitrary spin. 1. The boson case, Phys. Rev. D 9 (1974) 898 [inSPIRE].

[56] L.P.S. Singh and C.R. Hagen, Lagrangian formulation for arbitrary spin. 2. The fermion case, Phys. Rev. D 9 (1974) 910 [inSPIRE].

[57] B. de Wit and D.Z. Freedman, Systematics of Higher Spin Gauge Fields, Phys. Rev. D 21 (1980) 358 [InSPIRE].

[58] X. Bekaert and N. Boulanger, On geometric equations and duality for free higher spins, Phys. Lett. B 561 (2003) 183 [hep-th/0301243] [INSPIRE].

[59] X. Bekaert and N. Boulanger, Tensor gauge fields in arbitrary representations of $G L(D, R)$. II. Quadratic actions, Commun. Math. Phys. 271 (2007) 723 [hep-th/0606198] [InSPIRE]. 
[60] R. Jackiw and S. Templeton, How Superrenormalizable Interactions Cure their Infrared Divergences, Phys. Rev. D 23 (1981) 2291 [INSPIRE].

[61] L. Parker and D. Toms, Quantum Field Theory in Curved Spacetime, Cambridge Monographs on Mathematical Physics, Cambridge University Press, Cambridge U.K. (2009).

[62] E.E. Boos and A.I. Davydychev, A Method of evaluating massive Feynman integrals, Theor. Math. Phys. 89 (1991) 1052 [Teor. Mat. Fiz. 89 (1991) 56] [InSPIRE].

[63] A.I. Davydychev, A Simple formula for reducing Feynman diagrams to scalar integrals, Phys. Lett. B 263 (1991) 107 [INSPIRE].

[64] A.I. Davydychev. Recursive algorithm of evaluating vertex type Feynman integrals, J. Phys. A 25 (1992) 5587.

[65] B.L. de Souza, https://github.com/blimasouza/DavyFeyn.

[66] D. Anselmi, Higher spin current multiplets in operator product expansions, Class. Quant. Grav. 17 (2000) 1383 [hep-th/9906167] [INSPIRE]. 